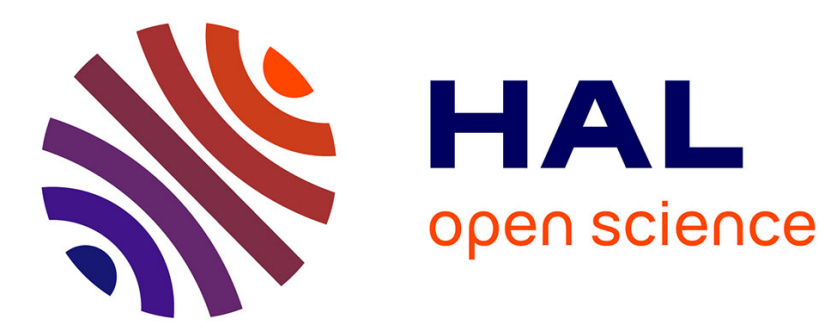

\title{
La pause méridienne: un facteur clé de l'évolution de la mobilité en France depuis 35 ans
}

\author{
Jean Paul Hubert, Joël Meissonnier, Jean Loup Madre, Sophie Roux
}

\section{To cite this version:}

Jean Paul Hubert, Joël Meissonnier, Jean Loup Madre, Sophie Roux. La pause méridienne: un facteur clé de l'évolution de la mobilité en France depuis 35 ans. Économie et statistique - Economy and Statistics, 2013, 457, pp 35-56. hal-00851376

\section{HAL Id: hal-00851376 \\ https://hal.science/hal-00851376}

Submitted on 13 Aug 2013

HAL is a multi-disciplinary open access archive for the deposit and dissemination of scientific research documents, whether they are published or not. The documents may come from teaching and research institutions in France or abroad, or from public or private research centers.
L'archive ouverte pluridisciplinaire HAL, est destinée au dépôt et à la diffusion de documents scientifiques de niveau recherche, publiés ou non, émanant des établissements d'enseignement et de recherche français ou étrangers, des laboratoires publics ou privés. 
La pause méridienne : un facteur clé de l'évolution de la mobilité en France depuis 35 ans

\author{
Hubert Jean-Paul*, Madre Jean-Loup*, Meissonnier Joël**, Roux Sophie* \\ *Université Paris Est, IFSTTAR, DEST \\ ** ERA-MOB, CETE - NORD PICARDIE \\ Ecrire à : jean-paul.hubert@ifsttar.fr
}

Résumé

Les déplacements des actifs, élèves ou étudiants quittant leur lieu de travail ou d'études pour déjeuner au domicile sont soumis à de fortes contraintes d'horaire et de distance, ainsi qu'à des arbitrages multiples au sein des ménages. L'évolution de cette pratique a aussi influencé fortement les indicateurs statistiques de la mobilité. En 1974, les déplacements réguliers lors de la pause méridienne représentaient $12 \%$ du total des déplacements réalisés par les Français et seulement $5 \%$ en 2008. La pratique du retour à midi pendant une journée de travail ou d'étude a décru à la même vitesse chez les actifs occupés et leurs enfants scolarisés. La féminisation de l'emploi et l'éloignement croissant des domiciles et des lieux de travail ou d'étude sont des causes importantes de cette disparition, mais celle-ci progresse plus vite que ces facteurs causaux. Ces déplacements méridiens sont néanmoins encore fréquents hors de la Région parisienne, même dans les grandes agglomérations. Ils concernent encore en 2008, 26\% de toutes les personnes se déplaçant pendant un jour de semaine.

Ce changement de comportement des actifs, élèves et étudiants explique $85 \%$ de la variation, observée pendant 34 ans, du nombre de déplacements par jour de semaine, de l'ensemble de la population. II devrait encore faire baisser l'indicateur de 0,04 à 0,06 déplacements par jour de 2008 à 2020. C'est aussi un facteur qui a stabilisé le temps passé quotidiennement en transport, en moyenne nationale. On observe néanmoins dans l'agglomération parisienne et, plus récemment, les principales métropoles régionales une autre baisse de la mobilité qui concerne des déplacements de plus longue portée et à d'autres tranches horaires. 


\section{Introduction}

Une pratique illustre la multiplicité des relations et des causalités qui agissent sur la mobilité et sa mesure par les enquêtes statistiques : rentrer du travail ou de l'école pour déjeuner multiplie par deux les déplacements liés à ces motifs, par rapport à la situation où l'on déjeune sur son lieu de travail ou d'étude, et cette pratique dépend de nombreux facteurs. Ainsi, l'emploi du temps sur le lieu de travail ou d'étude et la distance qui les sépare du domicile déterminent le temps disponible pour venir prendre un repas chez soi, après l'avoir éventuellement préparé, et retourner travailler l'après-midi. L'existence d'une offre de restauration collective, sa qualité et son coût, par rapport aux normes sociales définissant le repas de midi dans un pays ou une région, entrent aussi en ligne de compte. La mobilité des actifs employés, élèves ou étudiants autour de midi dépend donc d'arbitrages multiples entre les membres du ménage, voire entre collègues de travail.

Bien qu'en France le repas familial soit une spécificité culturelle restée très présente (Grignon et Grignon, 2004), les déjeuners sont de moins en moins souvent pris en famille au cours d'un jour de semaine; comme dans les autres pays d'Europe (Mestdag et Vandeweyer 2005). En 2006 : $49 \%$ des 3-10 ans, $66 \%$ des $11-14$ ans et $60 \%$ des $15-17$ ans prennent au moins trois de leurs déjeuners hebdomadaires au restaurant scolaire. Ces taux sont en progression globale de 8 points par rapport à 1999 (source : enquêtes INCA, AFSSA). Ce processus a été étudié dès la fin des années 1960 (Huu Thi 1970 ; Villeneuve 1974) et continue de l'être (Etiévant et al. 2010).

La baisse du nombre de déplacements quotidiens mesurée par les enquêtes a souvent été rapprochée de celle du nombre d'actifs ou d'enfants rentrant déjeuner à la maison. Madre et Maffre (1997) l'évoquent à propos de la variation observée (-7\%) entre les deux enquêtes nationales transport de 1982 et 1994. Plus récemment, l'agence d'urbanisme de Strasbourg note que la généralisation de la journée continue chez les actifs, élèves et étudiants, entre 1997 et 2009, a induit " mécaniquement » une baisse de 0,12 déplacement par jour de semaine pour l'ensemble de la population enquêtée (ADEUS 2010). Elle n'explique cependant qu'un cinquième de la baisse observée à Strasbourg, laquelle est élevée par rapport aux autres villes enquêtées récemment (données CERTU). Le processus d'externalisation du repas de midi suit une tendance de long terme même si le nombre de déplacements quotidiens peut varier pour bien d'autres raisons. Mais le " mécanisme » liant le lieu du déjeuner et la mobilité ne paraît guère avoir été approfondi, même lorsque la mobilité est envisagée du point de vue de la famille (Kaufmann et Flamm 2002 ; Le Breton 2005).

Le " déplacement » est un concept fondamental des enquêtes sur les transports et notamment des enquêtes nationales menées par l'INSEE pour le ministère chargé des transports. Il est défini comme le mouvement d'une personne utilisant un ou plusieurs modes de transport sur la voie publique, depuis un lieu de départ (origine) vers un lieu d'arrivée (destination) où elle va passer un certain temps pour réaliser une certaine activité (motif) (INSEE 2007). Le nombre de déplacements par jour est un indicateur statistique systématiquement présenté. Il est parfois vu comme révélateur de l'activité économique (Prud'homme 2010) mais sa signification est vague car l'objet " déplacement » est d'une extrême variété, même en se limitant à un voisinage habituel autour du domicile. A partir de l'enquête transport danoise qui est annuelle, Christensen (2004) montre que ce nombre est très dépendant de la méthodologie pour sa capacité à capter les déplacements les plus courts, à compter les 
journées réellement sans déplacements et à contacter les personnes qui se déplacent beaucoup. Et en effet, la série constituée par le Department for transport anglais de 1975 à 2011 - la plus riche collection d'enquêtes transport existant à un niveau national avec celle des Pays-Bas - montre de brusques sauts qu'il faut mettre en rapport avec les variations méthodologiques de la production des données (Anderson et al. 2009).

Comprendre comment varie le nombre de déplacements quotidiens nous paraît néanmoins important et cette évolution a dû être affectée par celle de la mobilité de milieu de journée entre le domicile et les lieux de travail ou d'étude. Nous nous proposons donc d'examiner le sous-ensemble relativement homogène des déplacements produits par la mobilité de la pause méridienne au niveau national, depuis les années 1970.

Cette mobilité est habituellement considérée dans la catégorie d'analyse « mobilité régulière et contrainte » car les lieux, les horaires des déplacements et leur fréquence au cours de l'année sont fixés, soit par des choix de long terme au sein du ménage, soit par un employeur ou un établissement d'enseignement. La quasi-totalité des élèves et étudiants et $80 \%$ des actifs travaillent dans un lieu fixe distinct du domicile, tandis que $8 \%$ des actifs travaillent à domicile et $12 \%$ en des lieux variables (François 2010). C'est cette mobilité régulière et contrainte qui engendre les phénomènes de pointe du matin, de midi et du soir. Or la pointe de midi s'est considérablement émoussée entre 1974 à 2008. Le pourcentage de déplacements commencés entre $11 \mathrm{~h} 30$ et $12 \mathrm{~h} 29$ un jour de semaine est passé de 11 à $8 \%$ du total de la journée (figure 1).

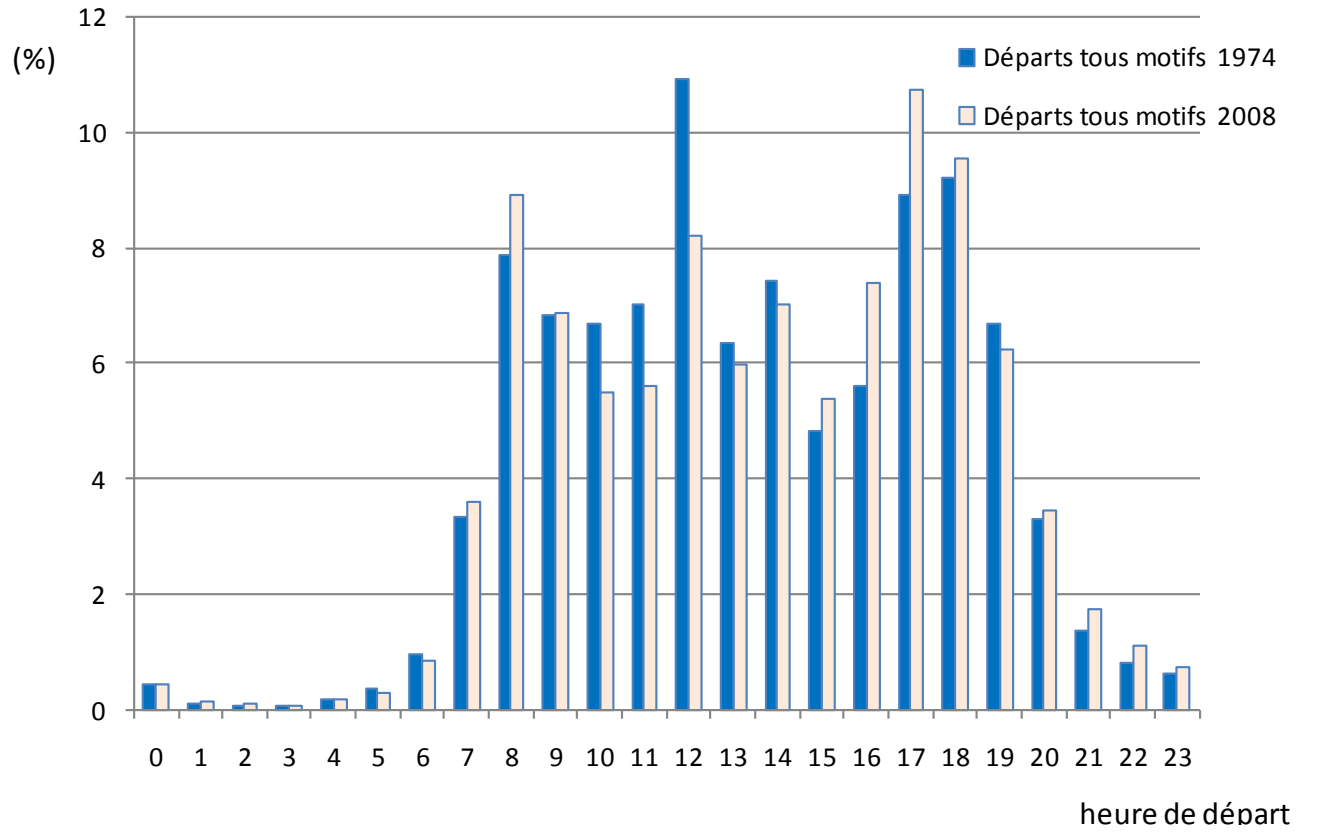

Figure 1 : Distribution horaire des départs de déplacements un jour de semaine en 1974 et 2008 Champ : déplacements de moins de $100 \mathrm{~km}$ un jour de semaine, sources : Insee, Enquête nationale transport 1974 et Insee, Inrets, SOeS : Enquête nationale transport et déplacements 2008 Lecture : 8\% des déplacements commencent entre 7h30et 8h29 en 1974 et 9\% en 2008

La « mobilité régulière et contrainte » recouvre donc deux types de déplacements dont l'évolution ne dépend pas des mêmes facteurs. Le premier et le dernier déplacement entre le domicile et le lieu de travail ou d'étude sont bien nécessaires à l'activité, sauf aménagement particulier ou télétravail, dans le cadre d'une durée légale aménageable par branche (RTT, 
notamment) par établissement (horaires d'équipe ou scolaires) ou individuellement, par recours au temps partiel. Mais la mobilité régulière lors de la pause méridienne n'est en rien nécessaire à l'activité de travail ou d'étude. Nous l'appelons « mobilité régulière supplémentaire » (MRS). Les individus se donnent une contrainte supplémentaire étant donné le temps - souvent très limité - dont ils disposent pour réaliser deux déplacements et un repas. Lorsque les contraintes s'accroissent - par exemple, du fait des impératifs professionnels, des conditions de trafic ou des distances domicile-travail - il est logique que ce supplément de mobilité régulière ne soit plus réalisé. C'est ce que nous allons vérifier en étudiant la MRS à partir des quatre enquêtes nationales menées par l'INSEE en 1973-74, 198182, 1993-94 et 2007-08, aujourd'hui exploitables. Notre objectif est d'en saisir certains déterminants et d'évaluer l'impact de la diminution de ce sous-ensemble dans l'évolution des statistiques sur la mobilité entre 1974 et 2008.

\section{Les données et leurs limites}

Les sources utilisées permettent d'analyser sur une longue période la mobilité de la pause méridienne à partir de deux jeux de données. Le premier porte sur l'organisation habituelle de la mobilité régulière. Une variable renseigne sur le nombre de navettes quotidiennes. A deux ou éventuellement trois navettes vers le lieu de travail ou d'études, nous supposons que l'individu rentre déjeuner à midi. Ce jeu de données est assis sur l'ensemble des membres du ménage de 6 ans et plus, à partir de l'enquête de 1981-82, mais réduit à l'ensemble des actifs et seulement un enfant scolarisé en 2007-08. II permet de mettre en perspective les habitudes des conjoints et des enfants.

Le second jeu de données rassemble les déplacements réalisés pendant une ou plusieurs journées précédant l'interview. Une seule personne du ménage a été interrogée. L'échantillon des journées est moins fourni que celui des navettes. II comporte plus d'alea mais les informations sont moins de nature déclaratives, ce qui peut expliquer certains écarts entre les traitements. Dans ce second jeu, dit de la " mobilité locale », nous ne considérons que les journées avec déplacements. Les variations du taux de journées sans mobilité nous paraissent peu liées a priori avec le comportement à la pause méridienne et, surtout, dépendre encore davantage des changements de méthode de collecte ou de traitement de la non-réponse, qui affectent la mesure de l'immobilité (Madre et al. 2007). Les quatre enquêtes françaises, bien que conçues dans la perspective d'une série statistique longue, ont connu d'assez lourdes variations de méthodologie, (Armoogum et al. 2010 et encadré 1 ) Les taux de journées sans déplacement sont néanmoins relativement proches pour les jours de semaine : entre $15,6 \%$ et $17,3 \%$ pour l'ensemble de la population de 6 ans et plus.

Si les sources utilisées permettent bien d'analyser la MRS, elles sont très lacunaires pour cerner l'objet qui peu à peu la remplace, à savoir la journée continue sur le lieu de travail, l'école ou l'établissement supérieur. Qu'en est-il de l'organisation du temps dans le cadre de ces journées et de son évolution, de la possibilité de se restaurer, à quel prix et à quelle qualité ? Quelles sont les activités réalisées pendant la pause méridienne en dehors de l'alimentation ? La dynamique de la journée continue est le point aveugle de notre étude. L'utilisation des enquêtes emploi du temps permettrait sans doute d'éclairer ces questions.

Dans la première partie, nous analysons les facteurs explicatifs de la mobilité régulière supplémentaire à partir des données sur les navettes des trois dernières enquêtes transport. 
Cette analyse permet de dégager les facteurs situationnels conditionnant concrètement la possibilité pour un individu de revenir manger au domicile et d'évaluer en outre un effet de période qui dénoterait davantage d'une évolution des conditions de la journée continue ou des mentalités. En deuxième partie, nous replaçons ces résultats dans l'évolution des indicateurs moyens de la mobilité locale observés de 1974 à 2008 pour un jour de semaine (nombre de déplacements, temps passé en transport et distance parcourue). Nous nous focalisons sur le sous-ensemble de déplacements à destination ou au départ du domicile, réalisés en milieu de journée par des actifs occupés, des élèves ou des étudiants présents sur leur lieu de travail en matinée et dans l'après-midi. Enfin, dans la troisième partie, nous extrapolons les tendances observées sur l'évolution de la mobilité de la pause méridienne. Des modèles aident à anticiper l'effet de la baisse tendancielle de cette pratique sur l'évolution future du nombre de déplacements global.

Insérer encadré 1 : Quelques questions de comparabilité entre les enquêtes nationales transports

\section{L'évolution des pratiques et de leurs facteurs explicatifs}

Les contraintes pesant sur les déplacements autour de midi

Au cours d'une évolution de près d'un siècle, la répartition rigide des rôles au sein du couple a fait place à une plus grande négociation pour le partage des tâches au sein d'une famille plus " relationnelle » (Singly 1996), composée plus tardivement dans le cycle de vie et sujette à décomposition et recompositions éventuelles - ce dont témoigne le doublement du nombre de célibataires actifs d'après les enquêtes de 1974 et 2008. L'ancienne répartition des rôles est représentée par le modèle familial de "Monsieur Gagne-Pain » (Poilot-Rocaboy et Kergoat, 2006) instauré au début du XXème siècle. A ce modèle correspondait une mobilité très différenciée des époux et notamment pendant la pause méridienne qui interrompt le travail du mari ou des enfants. Celle-ci est assurée dans le cadre des activités domestiques de l'épouse, lesquelles comprennent aussi des sorties pour les achats alimentaires.

Avec l'accès croissant des femmes à l'emploi et les revendications pour l'évolution des mœurs, les années 1970 voient le rejet de ce modèle. En 1975, les ménages à couple bi-actif sont déjà majoritaires (52\%; Ravel 2007). En 1981 néanmoins, selon l'enquête « vie familiale et vie professionnelle » de l'INED (Fagnani 1986), 54\% des mères salariées en Province rentrent déjeuner chez elles; elles ne sont que $27 \%$ dans l'agglomération parisienne, où leurs emplois sont aussi globalement plus qualifiés et leurs trajets domicile-travail plus longs. Si le travail féminin se généralise dans les années 1990 , les femmes assument encore « $80 \%$ du noyau dur » des tâches domestiques (Dumontier et Pan Ké Shon 1999) et les deux tiers de l'activité parentale (Algava 2002). Toutefois, cette activité domestique se concentre en soirée, le weekend ou les jours non travaillés en cas d'emploi à temps partiel. Les femmes aspirent logiquement à un allègement des contraintes dans leurs emplois du temps et leur désengagement dans l'organisation des repas de midi pendant la semaine semble avoir, plus facilement que d'autres, fait l'objet d'un compromis.

Cette évolution des relations dans les couples n'est pas séparable de celle de leur habitat ni des transformations des territoires, également sensibles en France à partir des années 1970. Un autre modèle se répand : celui du logement individuel en banlieue ou périphérie des villes, pour des ménages accédant à la propriété et ayant besoin de ce fait de deux salaires et de deux voitures (Merlin 2009). 
Au bilan de cette période, la distance moyenne entre domicile et lieu de travail fixe est passée de $6 \mathrm{~km}$ en 1967 (Villeneuve 1970) à 7,6km en 1974, 9km en 1982, 12km en 1994 et 14,7km en 2008 (Hivert et Orfeuil 1989, François 2010) pour un temps de trajet quasi constant de 20 à 21 minutes entre 1967 et 1994 mais passant à 23 minutes en 2008. La distance aux services et aux commerces aussi a augmenté (Gallez et al. 1997, Hilal 2007, Hubert et Delisle 2010). En outre, le lieu de travail est plus souvent variable (Crague 2003) ainsi que les horaires de travail et d'étude et la possibilité pour les membres de la famille de se retrouver à différentes plages horaires (Lesnard 2009).

Les moyens de transport médiatisent le rapport des individus au territoire et leur usage est bouleversé (tableau 1). La part des déplacements à pied ou en deux-roues s'effondre.

L'accessibilité aux lieux passe désormais par une technologie moderne et coûteuse jusque-là réservée aux plus grandes villes ou aux ménages aisés. Elle assure en 2008 95\% des distances parcourues. Mais malgré la voiture qui augmente la vitesse du transport, le retour au domicile est de moins en moins souvent possible pendant les pauses méridiennes des actifs occupés, élèves ou étudiants.

\begin{tabular}{|c|c|c|c|c|c|c|c|c|c|}
\hline Année & \multicolumn{2}{|c|}{$\begin{array}{l}\text { Déplacements. } \\
\text { (nombre /jour) }\end{array}$} & $\begin{array}{l}\text { Distance } \\
\text { parcourue } \\
\text { (km/jour) }\end{array}$ & $\begin{array}{l}\text { Temps de } \\
\text { transport } \\
\text { (mn/jour) }\end{array}$ & $\begin{array}{l}\text { Présence } \\
\text { domicile } \\
11-15 \mathrm{~h}(\%)\end{array}$ & \multicolumn{2}{|c|}{$\begin{array}{l}\text { Achats ou } \\
\text { Restaurant/Café } \\
11-15 \mathrm{~h}(\%)\end{array}$} & \multicolumn{2}{|c|}{$\begin{array}{l}\text { Seulement } \\
\text { Restaurant/Café } \\
11-15 \mathrm{~h}(\%)\end{array}$} \\
\hline 1974 & & 4,15 & 18,1 & 63 & 59 & & 19 & & 7 \\
\hline 1982 & & 4,12 & 21,6 & 68 & 55 & & 17 & & 5 \\
\hline 1994 & & 3,87 & 28,0 & 67 & 52 & & 16 & & 3 \\
\hline 2008 & & 3,81 & 31,0 & 70 & 47 & & 11 & & 4 \\
\hline \multirow[t]{2}{*}{ Année } & \multicolumn{4}{|c|}{ Partage en nombre de déplacements } & \multicolumn{5}{|c|}{ Partage en distance parcourue } \\
\hline & $\begin{array}{l}\text { Marche } \\
(\%)\end{array}$ & \begin{tabular}{|l|}
$\begin{array}{l}\text { 2Roues } \\
(\%)\end{array}$ \\
\end{tabular} & VP (\%) & TC (\%) & $\begin{array}{l}\text { Marche } \\
(\%)\end{array}$ & $\begin{array}{l}2 \text { Roues } \\
(\%)\end{array}$ & & $\mathrm{JP}(\%)$ & $\mathrm{TC}(\%)$ \\
\hline 1974 & 45 & 12 & 34 & 9 & 6 & 8 & & 63 & 23 \\
\hline 1982 & 34 & 9 & 49 & 8 & 4 & 5 & & 73 & 18 \\
\hline 1994 & 23 & 4 & 64 & 9 & 2 & 2 & & 82 & 14 \\
\hline 2008 & 22 & 4 & 65 & 9 & 2 & 3 & & 83 & 12 \\
\hline
\end{tabular}

Tableau 1 : Indicateurs de la mobilité quotidienne, de la pause méridienne et du partage modal pour l'ensemble de la population 1974-2008

Champ : déplacements de moins de $100 \mathrm{~km}$ un jour de semaine. Sources : Insee, Enquête nationale transport 1974, Insee-Inrets, Enquête sur les Transports 1982, Enquête Transport 1994 et Insee, Inrets, SOeS : Enquête nationale transport et déplacements 2008

L'organisation d'un déjeuner routinier à la maison calé sur les pauses du travail ou des études ne va plus de soi. La pause est ainsi devenue majoritairement extra-familiale, réalisée entre camarades de classes, collègues ou seul, de moins en moins au domicile. Le repas est souvent pris au lieu de travail : à la cantine, en emportant casse-croûte, tartines, gamelle à réchauffer sur place. Actifs et étudiants peuvent également se rendre dans un restaurant, une sandwicherie ou bien acheter de quoi manger dans un commerce alimentaire. Encore faut-il disposer de ces services à proximité suffisante. Près de la moitié des emplois est située hors des villes centres (Talbot 2001) souvent dans des zones d'activités dépourvues de ce genre d'aménités. On ne saurait donc oublier que pendant la période étudiée, les différents secteurs de la restauration - collective, traditionnelle ou rapide - ont aussi connu des bouleversements intenses. La qualité ainsi que l'image du repas ou des aliments pris sur le lieu de travail ou d'étude ont changé et fait l'objet de nombreux débats. La dynamique propre au modèle de la 
journée continue fait aussi partie des facteurs expliquant la disparition de la mobilité vers le domicile pendant la pause méridienne mais sort du cadre de notre étude.

\section{Les mêmes facteurs expliquent le retour à midi mais ils se produisent moins souvent}

Une analyse par régression logistique mesure et ordonne l'importance des facteurs pouvant expliquer le retour habituel au domicile pour déjeuner en 1982, 1994 et 2008. La méthode a aussi été appliquée par François (2010) mais seulement sur les données 2008 et sans tenir compte du comportement des autres membres du ménage ni de la catégorie sociale.

Entre 1982, 1994 et 2008, la proportion de navettes multiples passe de : 52\%, 43\% à 31\% pour les élèves et étudiants ; respectivement $43 \%, 37 \%$ et $26 \%$ pour les actifs employés. Malgré le renouvellement de la population active et, plus encore, scolaire au cours de ces 26 ans, les facteurs expliquant les navettes multiples restent les mêmes et interviennent dans le même ordre d'importance (encadré 2). Ce sont d'abord des variables liées à l'organisation des trajets ou à la synchronisation des pauses méridiennes, puis celles liées à la localisation résidentielle ou à la position sociale (tableaux 2 et 3 ). Ainsi, les principaux paramètres explicatifs s'appliquent soit aux individus (âge, mode de transport, activité des femmes dans un contexte monoparental ou de double activité des conjoints) soit aux ménages (synchronisation des comportements entre conjoints et enfants), étant entendu que ces comportements résultent d'arbitrages au sein du ménage, voire avec des personnes extérieures ou sorties du ménage.

La durée de trajet est un paramètre critique. A chaque enquête, plus de $90 \%$ des individus faisant deux navettes quotidiennes ont un temps de trajet inférieur ou égal à 20 minutes. Mais cette variable trouble l'analyse car elle est en partie déterminée par d'autres, voire parfois dépendante de la variable à expliquer. Ainsi, pour les élèves et étudiants, le changement d'école et de cycle d'étude diminue la probabilité de rentrer à midi. La zone de recrutement des établissements est à chaque fois plus peuplée, et plus la densité du territoire est faible, plus la distance au domicile s'en trouve augmentée. La durée de trajet dépend donc de l'âge, de la zone de résidence et du mode de transport employé. Mais dans une régression logistique, elle contribue davantage que les variables dont elle dépend. Pour les actifs occupés, la durée de trajet dépend du mode de transport, de la zone de résidence, du genre et de la catégorie sociale. Fagnani (1986) montre qu'au début des années 1980, les mères peu qualifiées ont eu souvent tendance à chercher un emploi proche, à pied, de leur domicile et qu'elles y déjeunaient plus souvent en semaine. Même si la décision de déjeuner à la maison est finalement déterminée par la durée du trajet, nous préférons la retirer du modèle logistique au profit des variables qui l'influencent. L'ajustement du modèle reste très satisfaisant.

Insérer : Encadré 2 : Commentaire de l'analyse par régression logistique

La baisse de deux cinquièmes environ de la pratique du retour à midi sur cette période s'explique par la rareté croissante des conditions favorables, mais pas seulement. Ainsi entre 1982 et 2008, la part des conjoints d'un actif occupé ne travaillant pas ou rentrant à midi de son travail ne passe que de $48 \%$ à $41 \%$. Celle des actifs occupés travaillant habituellement à 21 minutes ou plus de leur domicile passe de $23 \%$ à $35 \%$. Ils ne sont plus que $10 \%$ à faire le trajet 
à pied en 2008 contre $18 \%$ en 1982 . Concernant les élèves et étudiants, ils sont $53 \%$ à aller étudier à pied ou en deux-roues en 1982 et 42\% en 2008. En 1982, 29\% ont au moins un parent actif rentrant à midi, $24 \%$ n'en ont aucun, contre $23 \%$ et $29 \%$ respectivement en 2008 . Ces évolutions sont moins spectaculaires que la baisse du retour à midi. Le changement de comportement paraît anticiper l'évolution des contraintes individuelles d'emploi du temps.

Cependant, l'importance des variables de synchronisation avec les enfants et avec les conjoints dans les modèles logistiques implique que les comportements des parents actifs et des enfants élèves - plus que des étudiants - se déterminent l'un l'autre et évoluent parallèlement. Bien des enfants ne mangent chez eux à midi que parce qu'un ou les deux parents le font, ce qui est plus facile si l'un ne travaille pas. Réciproquement, des actifs rentrent manger à midi pour faire déjeuner leurs enfants scolarisés.

La similitude des régressions logistiques pour chaque enquête permet d'en réaliser une commune aux trois afin de mesurer l'effet de la période, c'est-à-dire : le différentiel de probabilité de faire deux navettes par jour en 1982 ou en 2008, par rapport à la référence 1994, toutes choses égales d'ailleurs. Cet estimateur montre que le comportement concernant les navettes évolue plus vite que les paramètres qui lui sont le plus corrélés et que la tendance s'est nettement accélérée après 1994. Placé dans les mêmes conditions dans son ménage et vis-à-vis de son lieu de travail, la probabilité pour un actif occupé d'effectuer deux navettes quotidiennes était ainsi de 3,0 points supérieure en 1982, par rapport à 1994 et 11,1 points inférieure en 2008. Pour un élève ou étudiant, les différentiels sont respectivement de 1,4 en plus et 11,8 en moins. Ces chiffres illustrent le parallélisme des trajectoires des parents et des enfants.

Au cours du temps, la mobilité entre le lieu de travail ou d'étude fixe et le domicile pour y déjeuner se fait donc de plus en plus rare. De fait, la mobilité régulière supplémentaire disparaît irréversiblement puisque des transformations profondes des familles et du territoire réduisent les conditions qui la rendent possible et qu'en outre, ce comportement requiert en fait la participation de plusieurs membres du ménage, ce qui en accroît la rigidité.

Tableaux 2 et 3 chacun sur une page. 


\begin{tabular}{|c|c|c|c|c|c|c|c|c|c|}
\hline Paramètre & Modalité & \multicolumn{2}{|c|}{$\begin{array}{l}\text { Ecart proba- } \\
\text { bilité } 1982\end{array}$} & \multicolumn{2}{|c|}{$\begin{array}{l}\text { Ecart proba- } \\
\text { bilité } 1994\end{array}$} & \multicolumn{2}{|c|}{$\begin{array}{l}\text { Ecart proba- } \\
\text { bilité } 2008\end{array}$} & \multicolumn{2}{|c|}{$\begin{array}{l}\text { Ecart proba. } 3 \\
\text { enquêtes }\end{array}$} \\
\hline \multicolumn{2}{|l|}{ Constante } & 38,5 & $* *$ & 35,5 & $* * *$ & 25,0 & $* * *$ & 35,9 & $* * *$ \\
\hline \multirow{4}{*}{$\begin{array}{l}\text { Quartile de } \\
\text { revenu par UC }\end{array}$} & $1 \mathrm{er}$ & 2,2 & & $-2,2$ & & $-2,9$ & $*$ & $-1,0$ & \\
\hline & 2ème & (réf.) & - & (réf.) & _ & (réf.) & & (réf.) & _ \\
\hline & 3ème & 1,5 & & $-3,8$ & $* * *$ & $-5,4$ & $* * *$ & $-3,0$ & $* * *$ \\
\hline & 4ème & $-3,8$ & & $-7,9$ & $* * *$ & $-3,9$ & $* * *$ & $-5,5$ & $* * *$ \\
\hline \multirow{4}{*}{$\begin{array}{l}\text { zone de } \\
\text { résidence }\end{array}$} & rural + périurbain petites agglo & $-1,7$ & & 1,5 & & $-0,6$ & & $-0,3$ & \\
\hline & autre périurbain et multi-pol. & $-6,3$ & $* *$ & $-9,1$ & $* * *$ & $-6,7$ & $* * *$ & $-8,1$ & $* * *$ \\
\hline & Grandes agglos sauf Paris & (réf.) & & (réf.) & & (réf.) & & (réf.) & \\
\hline & agglomération Paris & $-16,7$ & $* * *$ & $-14,6$ & $* * *$ & $-15,4$ & $* * *$ & $-16,7$ & $* * *$ \\
\hline \multirow[t]{2}{*}{ Genre } & homme & $-4,5$ & $* *$ & $-4,2$ & $* * *$ & 0,1 & & $-3,2$ & $* * *$ \\
\hline & femme & (réf.) & - & (réf.) & _- & (réf.) & _ & (réf.) & _- \\
\hline \multirow{2}{*}{$\begin{array}{l}\text { Temps de } \\
\text { travail }\end{array}$} & complet & (réf.) & _- & (réf.) & _- & (réf.) & - & (réf.) & 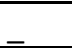 \\
\hline & partiel & $-23,4$ & $* * *$ & $-12,4$ & $* * *$ & $-1,5$ & & $-12,3$ & $* * *$ \\
\hline \multirow{4}{*}{$\begin{array}{l}\text { Synchronisation } \\
\text { avec le conjoint }\end{array}$} & pas de conjoint & 7,0 & $* *$ & 5,0 & $* * *$ & 11,2 & $* * *$ & 8,3 & $* * *$ \\
\hline & conjoint inactif & 16,5 & $* * *$ & 9,4 & $* * *$ & 13,0 & $* * *$ & 13,3 & $* * *$ \\
\hline & conjoint actif fait 2 navettes & 33,8 & $* * *$ & 31,0 & $* * *$ & 29,4 & $* * *$ & 31,9 & $* * *$ \\
\hline & conjoint actif fait 1 navette & (réf.) & & (réf.) & - & (réf.) & _- & (réf.) & \\
\hline \multirow{5}{*}{$\begin{array}{l}\text { Synchronisation } \\
\text { avec enfant(s) }\end{array}$} & pas d'enfant & $-2,2$ & & 5,5 & $* * *$ & 1,9 & & 1,7 & \\
\hline & un enfant fait une navette & (réf.) & $\ldots$ & (réf.) & $=$ & (réf.) & _ & (réf.) & - \\
\hline & un enfant fait 2 navettes & 21,7 & $* * *$ & 29,4 & $* * *$ & 15,3 & $* * *$ & 23,2 & $* * *$ \\
\hline & 2 enf,ou+ dont un à 2 navettes & $-5,0$ & & $-0,8$ & & $-1,3$ & & $-2,2$ & $*$ \\
\hline & 2 enf,ou+ dont 1 fait 1 navette & 14,6 & $* * *$ & 16,8 & $* * *$ & 7,8 & $* * *$ & 12,8 & $* * *$ \\
\hline \multirow{4}{*}{$\begin{array}{l}\text { Mode de } \\
\text { transport } \\
\text { domicile-travail }\end{array}$} & marche & 24,9 & $* * *$ & 22,2 & $* * *$ & 26,3 & $* * *$ & 24,3 & $* * *$ \\
\hline & vélo & 11,2 & $* * *$ & 8,5 & $* * *$ & 7,0 & $* * *$ & 9,3 & $* * *$ \\
\hline & voiture & (réf.) & $\ldots$ & (réf.) & $\ldots$ & (réf.) & - & (réf.) & _ \\
\hline & transports en commun & $-24,0$ & $* * *$ & $-20,1$ & $* * *$ & $-14,5$ & $* * *$ & $-21,0$ & $* * *$ \\
\hline \multirow[t]{5}{*}{ Classe d'âge } & $15-26$ ans & 1,2 & & 5,0 & $* * *$ & $-2,2$ & & 1,5 & \\
\hline & $27-36$ ans & $-2,5$ & & $-1,4$ & & $-4,6$ & $* * *$ & $-2,9$ & $* * *$ \\
\hline & $37-46$ ans & (réf.) & - & (réf.) & $\ldots$ & (réf.) & $\ldots$ & (réf.) & $\ldots$ \\
\hline & 47-56 ans & 3,2 & & 5,6 & $* * *$ & $-0,2$ & & 2,9 & $* *$ \\
\hline & 57 ans et plus & 12,5 & $* * *$ & 11,6 & $* * *$ & 0,3 & & 8,4 & $* * *$ \\
\hline \multirow[t]{4}{*}{ CS de l'actif } & agriculteurs & 40,0 & $* * *$ & 27,2 & $* * *$ & 49,1 & $* * *$ & 39,4 & $* * *$ \\
\hline & artisans & 22,7 & $* * *$ & 15,1 & $* * *$ & 21,1 & $* * *$ & 20,0 & $* * *$ \\
\hline & employés, prof inter. cadres & (réf.) & - & (réf.) & _ & (réf.) & $\ldots$ & (réf.) & $\ldots$ \\
\hline & ouvriers & $-3,8$ & $*$ & $-3,9$ & $* * *$ & $-3,8$ & $* * *$ & $-3,9$ & $* * *$ \\
\hline \multirow{2}{*}{$\begin{array}{l}\text { Type de } \\
\text { résidence }\end{array}$} & individuel & (réf.) & - & (réf.) & - & (réf.) & 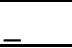 & (réf.) & - \\
\hline & collectif & $-0,5$ & & $-4,2$ & $* * *$ & $-4,0$ & $* * *$ & $-3,0$ & $* * *$ \\
\hline \multirow{3}{*}{$\begin{array}{l}\text { Année } \\
\text { d'enquête }\end{array}$} & 1982 & _ & _ & _ & _ & _ & - & 3,0 & $* * *$ \\
\hline & 1994 & & & & & & & (réf.) & _- \\
\hline & 2008 & - & & & & _- & & $-11,1$ & $* * *$ \\
\hline \multicolumn{2}{|c|}{ Concordance entre valeurs prédites et observées(\%) } & 79,1 & - & 73,9 & - & 74,5 & & 76,1 & - \\
\hline \multicolumn{2}{|c|}{ Taille de l'échantillon (individus) } & 3760 & & 12010 & & 16126 & & & \\
\hline
\end{tabular}

Code de significativité : ${ }^{* *}<=0,01 ;{ }^{* *}<=0,05 ;^{*}<=0,1$. Procédure SAS Surveylogistics

Tableau 2 : Régression logistique sur la probabilité de réaliser habituellement plusieurs navettes vers un lieu de travail fixe en 1982, 1994, 2008. Ecart de probabilité des variables explicatives

Champ : actifs occupés ayant un lieu de travail fixe à moins de $80 \mathrm{~km}$ du domicile déclarant 1 à 3 navettes

Sources : Insee-Inrets, Enquête sur les Transports 1982, Enquête Transport 1994 et Insee, Inrets, SOeS : Enquête nationale transport et déplacements 2008

Lecture : un actif qui va à son travail à pied a, toutes choses égales d'ailleurs, une probabilité de faire plusieurs navettes par jour supérieure à celle d'un actif utilisant une voiture, de 24,9 points en 1982, 22,2 en 1994 et 26,3 en 2008. "Grandes agglomérations » = pôle urbain d'une aire urbaine de 100.000 habitants et plus en 1999. 


\begin{tabular}{|c|c|c|c|c|c|c|c|c|c|}
\hline \multirow{2}{*}{$\begin{array}{l}\text { Paramètre } \\
\text { Constante }\end{array}$} & \multirow[t]{2}{*}{ Modalité } & \multicolumn{2}{|c|}{$\begin{array}{l}\text { Ecart proba- } \\
\text { bilité } 1982\end{array}$} & \multicolumn{2}{|c|}{$\begin{array}{l}\text { Ecart proba- } \\
\text { bilité } 1994 \\
\end{array}$} & \multicolumn{2}{|c|}{$\begin{array}{l}\text { Ecart proba- } \\
\text { bilité } 2008\end{array}$} & \multicolumn{2}{|c|}{$\begin{array}{l}\text { Ecart proba. } 3 \\
\text { enquêtes }\end{array}$} \\
\hline & & 21,4 & $* * *$ & 24,6 & $* * *$ & 30,3 & $* * *$ & 46,6 & \\
\hline \multirow{4}{*}{$\begin{array}{l}\text { zone de } \\
\text { résidence }\end{array}$} & Rural et périurbain & $-4,6$ & $* * *$ & $-5,5$ & $* * *$ & $-8,0$ & $* * *$ & $-11,2$ & $* * *$ \\
\hline & Grandes agglos sauf Paris & (réf.) & - & (réf.) & - & (réf.) & - & (réf.) & - \\
\hline & Banlieue Paris & $-8,7$ & $* * *$ & $-8,7$ & $* * *$ & $-10,1$ & $* * *$ & $-14,6$ & $* * *$ \\
\hline & Paris & 0,1 & & $-12,0$ & $* * *$ & $-12,7$ & $* * *$ & $-16,0$ & $* * *$ \\
\hline \multirow{4}{*}{$\begin{array}{l}\text { Quartile de } \\
\text { revenu par UC }\end{array}$} & $1 \mathrm{er}$ & $-4,5$ & $* *$ & $-3,4$ & $* *$ & 6,3 & $* *$ & 0,7 & \\
\hline & 2ème & (réf.) & - & (réf.) & - & (réf.) & - & (réf.) & - \\
\hline & 3ème & 1,3 & & $-2,5$ & & $-2,1$ & & $-4,5$ & $* * *$ \\
\hline & 4ème & 2,2 & & $-3,4$ & $*$ & $-1,3$ & & $-5,8$ & $* * *$ \\
\hline \multirow[t]{4}{*}{ CS du ménage } & Agriculteurs artisans & 7,0 & $* *$ & 2,3 & & 0,9 & & 3,5 & $*$ \\
\hline & Employés, prof inter. cadres & (réf.) & - & (réf.) & - & (réf.) & - & (réf.) & - \\
\hline & ouvriers & 3,3 & $*$ & 0,0 & & 10,2 & $* * *$ & 6,4 & $* * *$ \\
\hline & inactifs et retraités & $-3,2$ & & 12,1 & $* * *$ & 8,3 & $*$ & 1,5 & \\
\hline \multirow[t]{2}{*}{ Genre } & homme & 0,3 & & 0,1 & & 0,5 & & 0,2 & \\
\hline & femme & (réf.) & - & (réf.) & - & (réf.) & - & (réf.) & - \\
\hline \multirow{4}{*}{$\begin{array}{l}\text { Synchronisation } \\
\text { parentale }\end{array}$} & Parent(s) inactif(s) & 9,1 & $*$ & 4,1 & & 6,8 & $* *$ & $-21,8$ & $* * *$ \\
\hline & Parent(s) actif(s) disponible(s) & (réf.) & - & (réf.) & - & (réf.) & - & (réf.) & - \\
\hline & Un parent disponible, l'autre non & 14,8 & $* * *$ & 16,2 & $* * *$ & 21,8 & $* * *$ & 18,9 & $* * *$ \\
\hline & pas de parent disponible & $-10,0$ & $* * *$ & $-10,2$ & $* * *$ & $-16,9$ & $* * *$ & $-21,8$ & $* * *$ \\
\hline \multirow{2}{*}{$\begin{array}{l}\text { Synchronisation } \\
\text { avec autre } \\
\text { enfant }\end{array}$} & oui & 53,8 & $* * *$ & 53,5 & $* * *$ & s.o. & 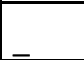 & s.o. & - \\
\hline & non & (réf.) & - & (réf.) & - & s.o. & - & s.o. & - \\
\hline \multirow{4}{*}{$\begin{array}{l}\text { Mode de } \\
\text { transport } \\
\text { domicile-école }\end{array}$} & marche & 31,9 & $* * *$ & 18,8 & $* * *$ & 23,3 & $* * *$ & 26,4 & $* * *$ \\
\hline & vélo & 17,3 & $* * *$ & 17,8 & $* * *$ & 16,4 & $* * *$ & 16,4 & $* * *$ \\
\hline & voiture & (réf.) & - & (réf.) & - & (réf.) & - & (réf.) & - \\
\hline & transports en commun & $-16,5$ & $* * *$ & $-16,1$ & $* * *$ & $-16,1$ & $* * *$ & $-23,4$ & $* * *$ \\
\hline \multirow[t]{4}{*}{ Classe d'âge } & 6-10 ans & 5,8 & $* * *$ & 4,3 & $* * *$ & 5,9 & $* *$ & 6,3 & $* * *$ \\
\hline & $11-15$ ans & (réf.) & _ & (réf.) & _ & (réf.) & _- & (réf.) & _ \\
\hline & $16-18$ ans & $-1,3$ & & $-1,6$ & & $-5,8$ & $*$ & $-3,7$ & $* *$ \\
\hline & $19-26$ ans & 12,1 & $* * *$ & $-3,5$ & $*$ & $-6,6$ & $* *$ & $-7,3$ & $* * *$ \\
\hline \multirow{2}{*}{$\begin{array}{l}\text { Type de } \\
\text { résidence }\end{array}$} & individuel & (réf.) & - & (réf.) & - & (réf.) & - & (réf.) & - \\
\hline & collectif & $-0,7$ & & 9,9 & $* * *$ & 1,5 & & 3,4 & $* *$ \\
\hline \multirow{3}{*}{$\begin{array}{l}\text { Année } \\
\text { d'enquête }\end{array}$} & 1982 & - & - & - & - & - & - & 1,4 & \\
\hline & 1994 & - & - & - & - & - & - & (réf.) & - \\
\hline & 2008 & - & - & - & - & - & - & $-11,8$ & $* * *$ \\
\hline \multicolumn{2}{|c|}{$\%$ paires concordantes } & 88,6 & & 86,7 & & 79,0 & & 80,8 & \\
\hline \multicolumn{2}{|c|}{ Taille de l'échantillon (individus) } & 3768 & & 7951 & & 5508 & & & \\
\hline
\end{tabular}

Code de significativité : $\left.{ }^{* *}<=0,01 ; * *<=0,05 ; *<=0,1\right)$. Procédure SAS Surveylogistics

Tableau 3 : Régression logistique sur la probabilité de réaliser habituellement plusieurs navettes vers un lieu d'études fixe en 1982, 1994, 2008. Ecart de probabilité des variables explicatives

Champ : élèves de 6 ans et plus et étudiants ayant un lieu d'étude fixe à moins de $80 \mathrm{~km}$ du domicile déclarant 1 à 3 navettes.

Sources : Insee-Inrets, Enquête sur les Transports 1982, Enquête Transport 1994 et Insee, Inrets, SOeS : Enquête nationale transport et déplacements 2008

Lecture : un enfant de 6 à 10 ans a, toutes choses égales d'ailleurs, une probabilité de faire plusieurs navettes par jour supérieure à celle d'un enfant de 11 à 15 ans, de 5,8 points en 1982, 4,3 en 1994 et 3,6 en 2008. 


\section{Influence de la pause méridienne sur les indicateurs de la mobilité pendant 35 ans}

\section{Mesurer le supplément de mobilité régulière dû à la pause méridienne}

Dans cette partie nous estimons les variations de la MRS à partir des déplacements réalisés entre le domicile et le lieu fixe de travail ou d'étude pendant une certaine plage horaire autour de midi. Les enquêtes de mobilité n'apportent pas en effet la réponse directe à la question " où déjeunent les individus ? ". Nous choisissons une plage relativement large : de $11 \mathrm{~h}$ à $15 \mathrm{~h}$ (bornes exclues) et supposons que les déplacements vers le domicile non liés au déjeuner sont en faible proportion et relativement constants au cours du temps. Avec ces conventions, en 1994, les temps de passage médians au domicile d'un actif occupé ou d'un élève ou étudiant présent à son lieu fixe et habituel de travail ou d'études à $11 \mathrm{~h}$ et $15 \mathrm{~h}$ (dans la suite, nous appellerons ce cas « journée complète ») sont de 65 et 75 minutes ; $5 \%$ des passages durent moins de 20 minutes et $10 \%$ plus de $1 \mathrm{~h} 45$.

La MRS est ici estimée par les déplacements vers le domicile commencés après $11 \mathrm{~h}$ et ceux partant du domicile et arrivant avant 15h, lors de " journées complètes » d'actifs, élèves ou étudiants. Pour les actifs, le taux de retour à midi estimé avec cette méthode est, à un point près, celui obtenu grâce aux navettes : 50\% en 1974, 43\% en 1982, 36\% en 1994, 25\% en 2008. II est systématiquement inférieur de 3 à 5 points pour les élèves et étudiants : $57 \%$ en 1974, $48 \%$ en 1982 , 38\% en $1994,27 \%$ en 2008 , mais les taux redeviennent tout à fait cohérents lorsqu'on retire les enfants de 6 à 10 ans. Les deux estimations divergent donc pour les enfants de l'école primaire. Est-ce dû au filtre de la journée complète dans l'observation de la mobilité, à une déclaration excessive du retour à midi par les parents qui décrivent la mobilité domicileétude ou à d'autres raisons ? La question reste ouverte mais n'a guère d'enjeu pour la suite.

\section{Conséquences de la diminution de la MRS}

D’environ 13,4 millions d'individus, actifs ou scolarisés, en 1974 à 16,2 millions en 2008, environ un tiers de la population totale, a réalisé une journée complète (tableau 4). En 1974, un peu plus de la moitié (53\%) de ces derniers effectuaient un déplacement vers leur domicile entre $11 \mathrm{~h}$ et $15 \mathrm{~h}$; un quart (26\%) en 2008. La part des ménages avec un seul actif dans le couple a fortement diminué au profit des ménages bi-actifs ou monoparentaux actifs. Or les retours au domicile des élèves ou étudiants pour déjeuner sont deux fois plus fréquents dans la première catégorie, en 1974 comme en 2008. 


\begin{tabular}{|c|c|c|c|c|c|c|c|c|c|c|}
\hline & \multicolumn{4}{|c|}{$\begin{array}{l}\text { Elève ou étudiant mobiles dans un } \\
\text { ménage... }\end{array}$} & \multicolumn{5}{|c|}{ actif occupé mobile avec ou sans enfants } & \multirow{2}{*}{$\begin{array}{l}\text { Ensemb } \\
\text { le des } \\
\text { mobiles }\end{array}$} \\
\hline & $\begin{array}{l}\text {...sans } \\
\text { actifs } \\
\text { occupé } \\
\text { s }\end{array}$ & $\begin{array}{l}\text {... avec } \\
\text { un seul } \\
\text { actif } \\
\text { dans le } \\
\text { couple }\end{array}$ & $\begin{array}{l}\text {... bi- } \\
\text { actif ou } \\
\text { mono- } \\
\text { parenta } \\
\text { l actif }\end{array}$ & tous & $\begin{array}{l}\text { célibata } \\
\text { ire }\end{array}$ & $\begin{array}{l}\text { un seul } \\
\text { actif } \\
\text { dans le } \\
\text { couple }\end{array}$ & $\begin{array}{l}\text { Ménag } \\
\text { e bi- } \\
\text { actif : } \\
\text { Homme }\end{array}$ & $\begin{array}{l}\text { Ménag } \\
\text { e bi- } \\
\text { actif : } \\
\text { Femme }\end{array}$ & tous & \\
\hline \multicolumn{11}{|c|}{ Présence au domicile à midi pendant une journée complète (\%) } \\
\hline 1974 & 49 & 67 & 43 & 57 & 37 & 57 & 53 & 48 & 51 & 53 \\
\hline 2008 & 32 & 49 & 21 & 27 & 22 & 27 & 26 & 26 & 25 & 26 \\
\hline \multicolumn{11}{|c|}{ Effectif par rapport à la population faisant une journée de travail ou d'études complète (\%) } \\
\hline 1974 & 1,6 & 20,0 & 12,8 & 34,4 & 10,0 & 24,0 & 17,0 & 14,6 & 65,6 & 100,0 \\
\hline 2008 & 2,5 & 6,2 & 22,1 & 30,8 & 16,0 & 8,1 & 25,7 & 19,4 & 69,2 & 100,0 \\
\hline \multicolumn{11}{|c|}{ Effectif par rapport à la population mobile totale (\%) } \\
\hline 1974 & 0,7 & 9,0 & 5,7 & 15,4 & 4,5 & 10,8 & 7,7 & 6,5 & 29,5 & 44,9 \\
\hline 2008 & 1,0 & 2,3 & 8,4 & 11,7 & 6,1 & 3,0 & 9,8 & 7,3 & 26,2 & 37,9 \\
\hline
\end{tabular}

Tableau 4 : Comportement de présence à midi pendant une journée de travail ou d'étude complète par type d'individu et de ménage en 1974-et 2008

Champ : Individus réalisant une journée de travail ou d'étude complète en semaine. Sources : Insee, Enquête nationale transport 1974, Insee, Inrets, SOeS : Enquête nationale transport et déplacements 2008

Lecture : les enfants de ménages bi-actifs en journée complète d'étude sont rentrés au domicile dans $43 \%$ des cas en 1974 et $21 \%$ en 2008 . Ils représentaient $13 \%$ des journées complètes de travail ou d'étude en $1974,22 \%$ en 2008 , respectivement $6 \%$ et $8 \%$ de la population mobile totale.

En séparant l'évolution de la MRS et le reste de la mobilité, en nombre de déplacements, nous constatons que cette dernière varie très peu en comparaison de la MRS et de la mobilité globale (tableau 5). Au niveau national, la tendance à la disparition du déjeuner à la maison a supprimé 0,29 déplacements par jour soit $85 \%$ de la baisse observée entre 1974 et 2008 pour cet indicateur. Elle explique donc cette baisse à condition que les déplacements domiciletravail/études de la pause méridienne ne soient pas remplacés par une mobilité vers d'autres lieux de restauration ou d'achats alimentaires, ce qui sera confirmé plus loin. Nous nous plaçons ainsi dans un modèle simple à deux populations (populations de journées individuelles types plutôt que d'individus, ceux-ci ayant en réalité plusieurs schémas d'activités) : d'une part, des actifs, élèves ou étudiants en journée complète sur un lieu de travail ou d'étude fixe (environ $30 \%$ des journées) dont le nombre quotidien de déplacements diminue et le temps passé en transport est stable, d'autre part : le reste des journées individuelles, où le nombre de déplacements est stable mais le temps de transport croît. Pour les deux populations, les distances s'allongent mais elles sont bien supérieures pour ceux qui ne pratiquent pas le retour à midi. La part de la MRS dans l'ensemble des déplacements est passée de $12 \%$ en 1974 à $10 \%$ en 1982, 7\% en 1994 et $5 \%$ en 2008. Quant aux distances parcourues, cette part est passée de $6 \%$ en 1974 à $3 \%$ en 2008 , et de $8 \%$ à $3 \%$ pour la durée passée en transport. 


\begin{tabular}{|c|c|c|c|c|}
\hline & 1974 & 1982 & 1994 & 2008 \\
\hline \multicolumn{5}{|c|}{ Nombre de déplacements moyen (France métropolitaine) } \\
\hline MRS & 0,48 & 0,40 & 0,29 & 0,19 \\
\hline Hors MRS & 3,67 & 3,72 & 3,58 & 3,62 \\
\hline Total & 4,15 & 4,12 & 3,87 & 3,81 \\
\hline Part de la MRS dans le total & $12 \%$ & $10 \%$ & $7 \%$ & $5 \%$ \\
\hline \multicolumn{5}{|c|}{ Durée moyennes en minutes (France métropolitaine) } \\
\hline MRS & 5,0 & 4,7 & 3,4 & 2,2 \\
\hline Hors MRS & 57,5 & 63,3 & 63,7 & 68,1 \\
\hline Total & 62,5 & 68,0 & 67,1 & 70,2 \\
\hline Part de la MRS dans le total & $8 \%$ & $7 \%$ & $5 \%$ & $3 \%$ \\
\hline \multicolumn{5}{|l|}{ Distance en km (France métropolitaine) } \\
\hline MRS & 1,1 & 1,0 & 1,2 & 0,8 \\
\hline hors MRS & 17,0 & 20,5 & 26,8 & 30,1 \\
\hline Total & 18,1 & 21,6 & 28,0 & 31,0 \\
\hline Part de la MRS dans le total & $6 \%$ & $5 \%$ & $4 \%$ & $3 \%$ \\
\hline \multicolumn{5}{|c|}{ Nombre de déplacements par zone de résidence } \\
\hline MRS agglo. Paris & 0,29 & 0,22 & 0,17 & 0,11 \\
\hline Hors MRS agglo. Paris & 3,95 & 3,75 & 3,42 & 3,28 \\
\hline Total agglo. Paris & 4,24 & 3,97 & 3,60 & 3,39 \\
\hline \% MRS dans total agglo. Paris & $7 \%$ & $5 \%$ & $5 \%$ & $3 \%$ \\
\hline MRS autres grandes agglos & 0,56 & 0,41 & 0,33 & 0,19 \\
\hline Hors MRS autres grandes agglos & 3,62 & 3,76 & 3,76 & 3,76 \\
\hline Total autres grandes agglos & 4,18 & 4,17 & 4,10 & 3,95 \\
\hline$\%$ MRS dans total autres grandes agglo. & $13 \%$ & $10 \%$ & $8 \%$ & $5 \%$ \\
\hline MRS reste France métropole & 0,50 & 0,45 & 0,30 & 0,21 \\
\hline Hors MRS reste France métropole & 3,59 & 3,69 & 3,53 & 3,64 \\
\hline Total reste France métropole & 4,09 & 4,14 & 3,83 & 3,86 \\
\hline$\%$ MRS dans total reste France métrop. & $12 \%$ & $11 \%$ & $8 \%$ & $6 \%$ \\
\hline
\end{tabular}

Tableau 5 : Evolution de la mobilité régulière supplémentaire et de la mobilité totale 1974-2008

« Grande agglomération » : pôle urbain d'une aire urbaine de 100.000 habitants et plus en 1999

Champ : déplacements de moins de $100 \mathrm{~km}$ un jour de semaine. Sources : Insee, Enquête nationale transport 1974, Insee-Inrets, Enquête sur les Transports 1982, Enquête Transport 1994 et Insee, Inrets, SOeS : Enquête nationale transport et déplacements 2008.

En nombre de déplacements, la part de la MRS est passée de $7 \%$ en 1974 à $3 \%$ en 2008 pour les résidents de l'agglomération parisienne, alors qu'elle passait de $12 / 13 \%$ à $5 / 6 \%$ en province. Le schéma dépend des zones de résidence des ménages. II s'applique mal dans l'agglomération parisienne où le niveau de la MRS est, au moins depuis 1974, bien plus faible que dans le reste du pays, à l'inverse des temps passés en transport. Le nombre de déplacements hors MRS y présente une nette tendance à la baisse. Les enquêtes ménages déplacements des plus grandes agglomérations montrent une baisse analogue depuis le tournant du siècle : Strasbourg (mentionnée en introduction), Lille, Lyon et Marseille (données CERTU). Cependant, la mobilité hors MRS de l'ensemble des grandes agglomérations affiche une grande stabilité entre les enquêtes nationales de1994 et 2008, tout comme dans les zones moins fortement urbanisées. 
Pour comparer l'évolution de la mobilité entre les populations qui réalisent une MRS et les autres, considérons trois classes de scolaires ou étudiants et autant d'actifs employés, selon qu'ils sont présents sur leur lieu de travail habituel pendant une journée complète (donc avec MRS potentielle), pendant une demi-journée (présence à $11 \mathrm{~h}$ ou $15 \mathrm{~h}$ ) et les autres (congés, lieu de travail variable, travail de nuit ou présence trop courte pour être relevée à $11 \mathrm{~h}$ ou à 15h). Complétons avec deux catégories d'inactifs ayant fini leurs études ou de chômeurs, selon qu'ils ont moins de 66 ans ou 66 et plus. La première classe contiendra les personnes inactives ou au chômage susceptibles de déjeuner avec un conjoint actif ou des enfants scolarisés, et de préparer le repas (tableau 6).

Nous pouvons affirmer que, pour les populations concernées, les déplacements de la MRS n'ont pas été remplacés par d'autres déplacements réalisés autour de midi et pouvant avoir une motivation alimentaire, au contraire. La fréquence de ces déplacements, qui n'excluent pas le retour au domicile, est restée très faible auprès des élèves ou étudiants pendant ces 34 ans : 3 à $4 \%$ des élèves étudiants en journées complètes, les plus âgés peut-on supposer, quittent leur établissement entre $11 \mathrm{~h}$ et $15 \mathrm{~h}$ pour acheter quelque chose ou consommer de la nourriture dans un café ou un restaurant. Le comportement était nettement plus fréquent pour les actifs employés en journée complète en 1974 : 19\% d'entre eux se déplaçaient pour ces motifs entre $11 \mathrm{~h}$ et $15 \mathrm{~h}$ ( $15 \%$ en retirant le motif achats). Leur part est tombée à, respectivement, $9 \%$ et $7 \%$ en 2008 . Elle a donc diminué de moitié, tout comme la fréquence du retour à la maison pour déjeuner. Le fait de ne plus manger à la maison entraîne logiquement la disparition de déplacements liés à l'achat des denrées à préparer, mais cela ne paraît pas suffisant. Il est probable que la pratique du déjeuner à l'extérieur hors du domicile a aussi diminué au profit d'un déjeuner pris sur le lieu de travail.

On a souvent vu dans la hausse de la mobilité (en se concentrant sur les déplacements mécanisés) un reflet de la croissance économique. Or la baisse de la MRS, qui est également corrélée à cette mécanisation signifie aussi une augmentation du PIB via le secteur de la restauration collective, publique ou privée.

En nombre de déplacements des individus mobiles, la baisse est continue dans les trois classes d'élèves ou étudiants. Cette baisse forte est plus régulière parmi ceux qui réalisent une journée complète (classe 3, concernée par la MRS), et parallèle à celle des actifs employés en journée complète (classe 6 ) où le nombre de déplacements moyen passe de 4,78 à 4,06 . Ce parallélisme manifeste encore la coordination de la MRS des adultes et des enfants. Dans les deux autres classes d'actifs employés, le nombre de déplacements quotidiens est stable. De même pour les inactifs et chômeurs mais leur effectif progresse parmi les personnes mobiles. Au bilan : les classes concernées par la MRS (de 45\% des effectifs mobiles en 1974 à 38\% en 2008) plus les deux autres classes d'élèves et étudiants (effectifs stables entre 9 et 10\%) contribuent le plus, de loin, à la baisse générale du nombre de déplacements. Un effet de moindre importance est dû aux variations d'effectifs des classes, en particulier au nombre croissant d'individus mobiles de plus de 65 ans qui réalisent en moyenne moins de déplacements que les autres. 


\begin{tabular}{|c|c|c|c|c|c|c|c|c|}
\hline $\begin{array}{l}\text { Classes } \\
\text { d'individus }\end{array}$ & Année & $\begin{array}{l}\text { Effectif } \\
(\%)\end{array}$ & $\begin{array}{l}\text { Déplct. } \\
\text { (nombre } \\
\text { /jour) }\end{array}$ & $\begin{array}{l}\text { Distance } \\
\text { parcourue } \\
\text { (km/jour) }\end{array}$ & $\begin{array}{l}\text { Temps de } \\
\text { transport } \\
\text { (mn/jour) }\end{array}$ & $\begin{array}{l}\text { Présence } \\
\text { domicile } \\
11-15 \text { h (\%) }\end{array}$ & $\begin{array}{l}\text { Achats ou } \\
\text { Rest./Café } \\
\text { 11-15h(\%) }\end{array}$ & $\begin{array}{l}\text { Seulement } \\
\text { Rest./Café } \\
11-15 \mathrm{~h}(\%)\end{array}$ \\
\hline \multicolumn{9}{|c|}{ Elèves et étudiants } \\
\hline \multirow{4}{*}{$\begin{array}{l}\text { Absents du } \\
\text { lieu d'étude } \\
\text { (1) }\end{array}$} & 1974 & 8 & 3,88 & 13,3 & 55 & 68 & 17 & 2 \\
\hline & 1982 & 6 & 3,57 & 13,5 & 52 & 63 & 15 & 3 \\
\hline & 1994 & 7 & 3,44 & 25,3 & 60 & 60 & 15 & 1 \\
\hline & 2008 & 6 & 3,44 & 24,6 & 62 & 53 & 10 & 3 \\
\hline \multirow{4}{*}{$\begin{array}{l}\text { Présents une } \\
\text { demi journée } \\
\text { (2) }\end{array}$} & 1974 & 2 & 4,35 & 17,1 & 72 & 82 & 14 & 6 \\
\hline & 1982 & 2 & 4,46 & 19,8 & 80 & 87 & 22 & 7 \\
\hline & 1994 & 3 & 3,63 & 22,4 & 76 & 79 & 4 & 1 \\
\hline & 2008 & 3 & 3,77 & 22,8 & 75 & 78 & 9 & 6 \\
\hline \multirow{4}{*}{$\begin{array}{l}\text { Présents deux } \\
\text { demi journées } \\
\text { ou : journée } \\
\text { complète (3) }\end{array}$} & 1974 & 15 & 4,03 & 8,3 & 49 & 57 & 6 & 3 \\
\hline & 1982 & 16 & 3,88 & 10,9 & 58 & 48 & 4 & 3 \\
\hline & 1994 & 14 & 3,62 & 15,5 & 58 & 38 & 3 & 3 \\
\hline & 2008 & 12 & 3,32 & 16,9 & 55 & 27 & 4 & 4 \\
\hline \multicolumn{9}{|l|}{ Actifs employés } \\
\hline \multirow{4}{*}{$\begin{array}{l}\text { Absents d'un } \\
\text { lieu de travail } \\
\text { fixe (4) }\end{array}$} & 1974 & 13 & 4,07 & 24,3 & 67 & 59 & 26 & 6 \\
\hline & 1982 & 11 & 3,92 & 26,9 & 69 & 50 & 25 & 4 \\
\hline & 1994 & 12 & 3,78 & 31,8 & 63 & 52 & 25 & 2 \\
\hline & 2008 & 14 & 4,09 & 36,0 & 74 & 56 & 15 & 5 \\
\hline \multirow{4}{*}{$\begin{array}{l}\text { Présents une } \\
\text { demi journée } \\
\text { (5) }\end{array}$} & 1974 & 8 & 4,22 & 26,7 & 76 & 79 & 15 & 6 \\
\hline & 1982 & 10 & 4,26 & 30,3 & 75 & 79 & 15 & 5 \\
\hline & 1994 & 8 & 4,01 & 37,1 & 77 & 78 & 12 & 3 \\
\hline & 2008 & 9 & 4,29 & 41,3 & 78 & 76 & 8 & 2 \\
\hline \multirow{4}{*}{$\begin{array}{l}\text { Présent deux } \\
\text { demi journées } \\
\text { ou : journée } \\
\text { complète (6) }\end{array}$} & 1974 & 30 & 4,78 & 26,2 & 77 & 51 & 19 & 15 \\
\hline & 1982 & 29 & 4,78 & 31,0 & 81 & 43 & 16 & 10 \\
\hline & 1994 & 26 & 4,40 & 39,5 & 81 & 36 & 10 & 7 \\
\hline & 2008 & 26 & 4,06 & 40,9 & 81 & 25 & 9 & 7 \\
\hline \multicolumn{9}{|c|}{ Inactifs et chômeurs } \\
\hline \multirow{4}{*}{$\begin{array}{l}\text { Age }<66 \text { ans } \\
\text { (7) }\end{array}$} & 1974 & 15 & 3,78 & 11,8 & 50 & 60 & 26 & 1 \\
\hline & 1982 & 16 & 3,98 & 16,1 & 63 & 60 & 25 & 3 \\
\hline & 1994 & 20 & 4,04 & 24,5 & 63 & 61 & 24 & 2 \\
\hline & 2008 & 18 & 3,86 & 27,9 & 68 & 56 & 14 & 2 \\
\hline \multirow{4}{*}{$\begin{array}{l}\text { Age }>65 \text { ans } \\
\text { (8) }\end{array}$} & 1974 & 9 & 3,13 & 7,0 & 47 & 59 & 23 & 3 \\
\hline & 1982 & 10 & 3,10 & 10,2 & 53 & 58 & 22 & 2 \\
\hline & 1994 & 10 & 2,95 & 15,2 & 53 & 60 & 26 & 2 \\
\hline & 2008 & 12 & 3,13 & 19,1 & 57 & 58 & 15 & 2 \\
\hline
\end{tabular}

Tableau 6 : Indicateurs de la mobilité quotidienne et pendant la pause méridienne par classes d'individus, 1974-2008

Champ : déplacements de moins de $100 \mathrm{~km}$ un jour de semaine. Sources : Insee, Enquête nationale transport 1974, Insee-Inrets, Enquête sur les Transports 1982, Enquête Transport 1994 et Insee, Inrets, SOeS : Enquête nationale transport et déplacements 2008 Lecture : parmi les actifs employés en journée complète (sur leur lieu de travail fixe) en 1994, 36\% étaient présents à leur domicile entre $11 \mathrm{~h}$ et $15 \mathrm{~h}$; pendant cette période, $10 \%$ ont réalisé un déplacement pour faire des achats ou se restaurer, dont $7 \%$ un déplacement pour se restaurer, seulement.

La disparition de la MRS est moins visible dans les autres dimensions de la mobilité de semaine. Le temps de parcours et la distance parcourues suivent en effet une tendance générale à la hausse, au contraire du nombre de déplacements. Cette évolution fait néanmoins comprendre que la stabilité moyenne du temps de transport quotidien des actifs occupés et 
des élèves étudiants, surtout depuis 1982 (81 minutes pour les actifs, 55 à 58 minutes pour les élèves et étudiants) masque une nette augmentation du-temps de trajet entre le domicile et les lieux de travail ou d'étude. Tout se passe comme si, en effet, deux allers-retours quotidiens étaient remplacés par un aller-retour deux fois plus long en durée. La motorisation croissante de la MRS, à l'instar du reste de la mobilité, explique pourquoi, avec moins de déplacements réalisés, la distance qu'elle représente dans la moyenne générale reste à peu près constante, autour de 1,0 km par jour rapporté à l'ensemble de la population mobile. La voiture permettant d'aller plus vite, le retour au domicile pour déjeuner est possible dans un rayon plus grand autour du domicile. Notons encore cette conséquence paradoxale : si les enfants expérimentent plus précocement un moment de socialisation et d'émancipation vis-à-vis des parents lors du repas de midi, leur transport vers l'école, où ils sont plus souvent passagers d'une voiture ou d'un car, est en revanche de moins en moins autonome et réduit qualitativement leur expérience du territoire.

\section{Un effet marginal sur l'évolution des parts modales et leur mesure}

Replacés dans l'échantillon des déplacements, représentatif de la mobilité locale des Français métropolitain en semaine, la MRS donne, mais de moins en moins, une importance double à des déplacements domicile-travail ou domicile-étude courts et réalisés plus souvent à pied ou en deux-roues et moins souvent en transport en commun. Dans une étude chronologique, la disparition de la MRS déforme donc les variations du partage modal par rapport à l'évolution du choix du mode déplacement par les individus. Qu'un actif dont la distance domicile travail est passée de $800 \mathrm{~m}$ à $8 \mathrm{~km}$ choisisse de prendre sa voiture au lieu de marcher et ce sont quatre déplacements à pied remplacés par deux en voiture. Les élèves, ou leurs parents, n'ont pas seulement choisi un mode de transport pour un autre, ils ont substitué un schéma à quatre déplacements par jour par un schéma à deux. La disparition de la MRS a donc accentué la baisse de la marche et, dans une moindre mesure celle des deux-roues dans les parts modales. La même remarque s'applique - faut-il le dire ? - à l'analyse de fréquence des motifs de déplacement, dont l'évolution biaise celle de la fréquence des activités de travail et d'études.

\section{Conséquence sur l'évolution future de la mobilité}

Au vu de ces tendances fortes et régulières observées depuis 1974, le comportement de retour à midi paraît raisonnablement prévisible à moyen terme, pour près d'un tiers des personnes mobiles un jour de semaine.

Commençons par extrapoler une simple régression linéaire des quatre points d'observations (tableau 7). Sur la période 1974-2008, le taux de retour à midi chez les élèves et étudiants réalisant une journée complète a baissé de 8 points par décennie. Au vu des taux de retour à midi encore observés en 2008 ( 25 à $30 \%$ ) nous supposons que la tendance peut continuer à ce rythme pendant encore une douzaine d'année. Le taux perdrait encore 7 à 12 points de pourcentage selon les zones ; il s'élèverait à $16 \%$ pour l'ensemble des élèves étudiants en 2020. Le même calcul d'interpolation est réalisé pour les actifs occupés et donne également pour résultat un taux de $16 \%$ et une baisse de 6 à 10 points selon les zones. 
Mais tout exercice de projection doit se baser sur la compréhension des tendances passées et il est utile d'approfondir le mécanisme grâce à un modèle " âge-cohorte " appliqué aux données sur la mobilité locale depuis 1974 des actifs occupés (Bussière et al. 1996 ; encadré 3). D'après les analyses logistiques, en effet, la probabilité de rentrer déjeuner à la maison dépend de facteurs liés au cycle de vie. La naissance des enfants peut signifier un déménagement vers un logement en périphérie allongeant le temps de transport vers le lieu de travail et abaissant la probabilité de rentrer à midi. Mais c'est leur scolarisation qui peut amener à l'organisation d'un déjeuner en famille pendant la pause méridienne, en relation avec une possible interruption du travail de la mère. La reprise éventuelle de son activité fait à nouveau baisser la probabilité de rentrer à midi pour son conjoint, ses enfants et elle-même de surcroît. La possible rupture du couple agit dans le même sens. Mais la probabilité de ces événements dépend de la génération, dont le modèle permet d'isoler l'effet. Nous avons également souligné que le niveau de la MRS dépendait du degré d'urbanisation, c'est pourquoi un modèle est réalisé pour chaque type de zone. Dans le modèle âge-cohorte, classes d'âges et générations avancent à un pas de dix ans. Chaque profil-type (lignes des classes d'âge du tableau 8) est stable entre 30 et 40 ans, décroît avant et après et remonte après la cinquantaine, pour les populations des grandes agglomérations. Les écarts entre générations sont réguliers et représentent une baisse générale de 7,5 points de pourcentage tous les dix ans ( 3 points l'agglomération de Paris et 9 pour le reste du territoire).

Les intervalles de temps entre les enquêtes ( 8,12 et 14 ans) étant du même ordre de durée que le cycle scolaire (10 à 15 ans) le modèle âge-cohorte est impraticable pour les élèves et étudiants mais nous pouvons considérer que leur évolution est parallèle à celle des actifs. Pour estimer le taux de retour à midi en 2020, le modèle âge-cohorte devrait être combiné à des prévisions démographiques par classe d'âge et par zone. En première hypothèse de distribution constante des classes d'âge par zone, nous pouvons approcher l'évolution 2010 à 2020 à partir du taux modélisé en 2010 moins un décalage de génération égal à la moyenne des trois derniers. On obtient : 17,2\% pour l'ensemble, 23,7\% dans les zones peu urbanisées, $16,5 \%$ dans les grandes agglomérations sauf Paris et 4,2\% dans l'agglomération parisienne ; légèrement au-dessus de l'interpolation linéaire. Considérons l'intervalle produit par les deux méthodes. Cette baisse se traduirait donc par la non-réalisation de deux déplacements par 7 à $10 \%$ des individus en journée complète de travail ou d'étude, estimés en gardant le taux 2008 à $36 \%$ de la population mobile (48 millions en 2008 , réévalués de $6,5 \%$ grâce aux projections en 2020 de l'INSEE) : 2,6 à 3,7 millions de déplacements retirés du supplément de mobilité régulière. Pour une population de l'ordre de 65 millions de personnes de 6 ans et plus dans un ménage ordinaire (base de sondage des enquêtes transport) cette disparition prévisible de la MRS représenterait donc, très grossièrement, 0,04 à 0,06 déplacement en moins par jour et par personne sur 12 ans.

Insérer Encadré 3 : Le modèle âge-cohorte 


\begin{tabular}{|c|c|c|c|c|c|}
\hline \multicolumn{2}{|c|}{ Passage au domicile entre $11 \mathrm{~h}$ et $15 \mathrm{~h}$} & \multirow{2}{*}{$\begin{array}{r}\text { Ensemble } \\
(\%)\end{array}$} & \multirow{2}{*}{$\begin{array}{r}\text { Zone } \\
\text { faiblement } \\
\text { urbanisée (\%) }\end{array}$} & \multirow{2}{*}{$\begin{array}{r}\text { Grandes } \\
\text { agglomérations } \\
\text { sauf Paris (\%) } \\
\end{array}$} & \multirow{2}{*}{$\begin{array}{r}\text { Agglomération } \\
\text { parisienne (\%) }\end{array}$} \\
\hline Date $\mathrm{m}$ & servation & & & & \\
\hline \multicolumn{6}{|c|}{ Elèves étudiants } \\
\hline \multicolumn{2}{|c|}{2007,7} & 27,5 & 25,8 & 32,2 & 24,0 \\
\hline \multicolumn{2}{|c|}{$\begin{array}{c}2020 \\
\text { (extrapolation selon modèle linéaire ) }\end{array}$} & 16,2 & 13,7 & 19,9 & 17,3 \\
\hline \multirow[t]{2}{*}{ te $=A^{*}($ annee -1970$)+B$} & A & $-0,00858$ & $-0,00844$ & $-0,00963$ & $-0,00669$ \\
\hline & B & 0,59081 & 0,55877 & 0,68091 & 0,5072 \\
\hline \multicolumn{6}{|c|}{ Actifs occupés } \\
\hline \multicolumn{2}{|c|}{2007,7} & 25,1 & 32,9 & 25,6 & 8,2 \\
\hline \multicolumn{2}{|c|}{$\begin{array}{c}2020 \text { (extrapolation selon modèle } \\
\text { linéaire " } t » \text { ) }\end{array}$} & 16,3 & 23,4 & 15,5 & 2,2 \\
\hline \multirow{2}{*}{$\begin{array}{c}\text { ta }=A *(\text { annee }- \\
1970)+B\end{array}$} & $A$ & $-0,00732$ & $-0,00828$ & $-0,00834$ & $-0,004$ \\
\hline & B & 0,52879 & 0,64811 & 0,57162 & 0,22161 \\
\hline
\end{tabular}

Tableau 7 : Taux observés et extrapolation de la probabilité de rentrer au domicile entre $11 \mathrm{~h}$ et $15 \mathrm{~h}$ d'un élève ou étudiant en journée complète d'étude

Champ : Elèves ou étudiants de 6 ans et plus présents à $11 \mathrm{~h}$ et $15 \mathrm{~h}$ sur leur lieu d'étude. Sources : Insee, Enquête nationale transport 1974, Insee-Inrets, Enquête sur les Transports 1982, Enquête Transport 1994 et Insee, Inrets, SOeS : Enquête nationale transport et déplacements 2008.

\begin{tabular}{|c|c|c|c|c|}
\hline Estimations & Ensemble & $\begin{array}{c}\text { Zone faiblement } \\
\text { urbanisée }\end{array}$ & $\begin{array}{c}\text { Grandes } \\
\text { agglomérations sauf } \\
\text { Paris }\end{array}$ & $\begin{array}{l}\text { Agglomération } \\
\text { parisienne }\end{array}$ \\
\hline$R^{2}$ & 0,414 & 0,514 & 0,448 & 0,173 \\
\hline \multicolumn{5}{|c|}{ Classe d'âge (cohorte de référence 1945-1955) } \\
\hline $16-25$ ans & $0,532 * * *$ & $0,650 * * *$ & $0,535 * * *$ & $0,247 * * *$ \\
\hline $26-35$ ans & $0,395 * * *$ & $0,491 * * *$ & $0,469 * * *$ & $0,131^{* * *}$ \\
\hline $36-45$ ans & $0,408 * * *$ & $0,504^{* * *}$ & $0,478 * * *$ & $0,122 * * *$ \\
\hline $46-55$ ans & $0,325 * * *$ & $0,453 * * *$ & $0,332 * * *$ & $0,080 * * *$ \\
\hline 56 ans et + & $0,357 * * *$ & $0,455^{* * *}$ & $0,381 * * *$ & $0,166 * * *$ \\
\hline \multicolumn{5}{|c|}{ Cohorte (année de naissance) } \\
\hline Avant 1925 & $0,190 * * *$ & $0,195 * * *$ & $0,278 * * *$ & $0,077 \quad * *$ \\
\hline 1925-1934 & $0,141 \quad * * *$ & $0,161 \quad * * *$ & $0,106 * * *$ & $0,106 * * *$ \\
\hline 1935-1944 & $0,068 \quad * * *$ & $0,062 \quad * *$ & $0,077 \quad * *$ & $0,063 * * *$ \\
\hline 1945-1954 & 0 (réf,) & 0 (réf,) & 0 (réf,) & 0 (réf,) \\
\hline 1955-1964 & $-0,051 \quad * * *$ & $-0,053 * *$ & $-0,132 \quad * * *$ & 0,017 \\
\hline 1965-1974 & $-0,154 \quad * * *$ & $-0,189 \quad * * *$ & $-0,171 \quad * * *$ & $-0,087 \quad * * *$ \\
\hline Après 1974 & $-0,230 * * *$ & $-0,270 * * *$ & $-0,268 * * *$ & $-0,095 * * *$ \\
\hline
\end{tabular}

Tableau 8 : Modèle âge-cohorte pour la probabilité de rentrer au domicile entre $11 \mathrm{~h}$ et $15 \mathrm{~h}$ d'un actif en journée complète de travail

Champ : actifs ayant un emploi, présents à $11 \mathrm{~h}$ et $15 \mathrm{~h}$ sur leur lieu de travail habituel

Sources : Insee, Enquête nationale transport 1974, Insee-Inrets, Enquête sur les Transports 1982, Enquête Transport 1994 et Insee, Inrets, SOeS : Enquête nationale transport et déplacements 2008.

Lecture : un individu en journée complète de travail sur son lieu habituel, âgé de 26 à 35 ans et né entre 1945 et 1954 (ou autrement dit : un individu d'environ 30 ans considéré autour de l'année 1980) a une probabilité égale à $39,5 \%$ de passer à son domicile entre $11 \mathrm{~h}$ et $15 \mathrm{~h}$. Pour un individu du même âge considéré autour de l'an 2000, et donc né entre 1965 et 1974, la probabilité a varié du coefficient de la génération et vaut : 0,395-0,154 = 24\%. En l'espace de vingt ans, la probabilité de passer au domicile pendant une journée complète de travail pour une personne de 30 ans à baissé de 15 points. 


\section{Conclusion}

L'évolution des indicateurs de base de la mobilité de semaine entre 1974 et 2008 montre que les transformations structurelles de la société française se sont cristallisées dans la disparition d'une pratique de mobilité : le retour au domicile pour déjeuner pendant une journée de travail ou d'étude. Cette pratique produit une classe de déplacements que nous avons appelée " mobilité régulière supplémentaire » (MRS) dont la part dans l'ensemble des déplacements des Français un jour de semaine a littéralement fondu, passant de $12 \%$ à $5 \%$ du total des déplacements en 34 ans. La mobilité régulière supplémentaire montre des niveaux différents entre l'agglomération parisienne et le reste de la France métropolitaine mais elle a régressé partout à la même vitesse. Cette diminution constante va se poursuivre à moyen terme.

Deux autres conclusions peuvent être tirées : sur l'évolution des comportements d'une part, sur l'interprétation des variations temporelles des indicateurs statistiques de la mobilité, d'autre part. L'étude peut aussi être prolongée dans deux directions au moins.

La disparition de la MRS explique la forte atténuation de la « pointe de midi » car la pratique du retour à midi a été remplacée par celle de la journée continue sur un lieu de travail ou d'étude plus éloigné du domicile. Aucune augmentation des déplacements pour achats ou consommation d'aliments entre $11 \mathrm{~h}$ et $15 \mathrm{~h}$ n'est observable de la part des actifs ou des élèves et étudiants pendant leurs journées de travail, au contraire. Le repas de midi étant de moins en moins familial, en France comme ailleurs en Europe, le déjeuner pèse significativement plus lourd sur le budget des ménages actifs et des familles, même s'il est subventionné par l'employeur ou les communes. Les transports pour aller travailler ou étudier sont également une charge croissante pour les ménages. Une bonne part de l'abandon d'un transport quasi gratuit au profit de déplacements mécanisés de plus en plus coûteux en temps et en argent est également liée au phénomène.

La disparition de la MRS ne doit jamais être négligée dans l'interprétation des variations temporelles des indicateurs de la mobilité, tout particulièrement lorsqu'on met l'accent sur le nombre moyen de déplacements quotidiens. Son évolution observée entre 1974 et 2008 provient presque en totalité de celle de la MRS. Les autres composantes de la mobilité ne suivent pas de tendances aussi claires et leurs oscillations se compensent à peu près. On observe néanmoins dans les plus grandes villes françaises une baisse de la mobilité qui concerne aussi ses autres dimensions (distances et temps quotidiens), ce que la MRS seule ne peut provoquer, et qui mérite des études spécifiques (Guidez et al., 2007). Soulignons alors le peu de signification du nombre de déplacements quotidien lorsqu'on le considère en moyenne générale au lieu de le décomposer selon des catégories de populations ayant des programmes d'activité homogènes. Ce nombre n'est pas un bon indicateur de la diversité des activités réalisées puisque certaines d'entre elles, à l'instar du travail et des études, sont massivement comptées deux fois. C'est pourquoi l'évolution de la MRS biaise aussi la comparaison temporelle de la fréquence des déplacements selon les modes et les motifs.

Mais la trajectoire temporelle de la MRS n'est pas autonome, loin s'en faut. L'augmentation générale des distances et des durées de transport entre le domicile et le lieu de travail ou d'études et la bi-activité croissante des couples sont les principaux facteurs explicatifs analysables à partir des données utilisées. Ils entraînent la désynchronisation des agendas individuels au sein des familles, affectent autant la relation entre parents qu'entre parents et enfants, et accroissent la pression sur l'emploi du temps des femmes. Or les repas pris à domicile par les actifs sont étroitement corrélés à ceux pris par les enfants scolarisés. Les deux 
pratiques diminuent donc de conserve. On peut aussi supposer que le modèle de la pause méridienne à domicile, voire simplement hors du lieu de travail, a perdu de son attractivité au profit de celui de la journée continue. Mais les enquêtes transport ne nous apportent pas d'autres informations. Comment est et sera pris le repas de midi : à table à plusieurs ou seul dans le bureau ? Plus généralement, à quoi est - ou sera - utilisé le temps de la pause méridienne : moment de pause socialisée, avec les collègues, avec des amis à distance via un réseau social du Web ; moment plus solitaire de loisir ou utilitaire, achats en magasin ou sur Internet ? La question tient à d'autres enjeux échappant à la statistique de la mobilité, comme l'évolution de la qualité de la vie au travail, des relations entre collègues, de l'hygiène alimentaire, de la gestion du temps au bureau...

Remarquons enfin que les phénomènes ayant déterminé ces changement d'organisation de la journée en semaine se sont produit a priori partout mais pas selon la même périodisation. Certains pays ou régions ont connu plus tôt que d'autres la déconcentration de la population urbaine, comme la Belgique, ou plus tard l'entrée massive des femmes dans le marché du travail, comme l'Espagne (source Eurostat). Les habitudes et les normes concernant le repas de midi n'étaient pas non plus les mêmes, par exemple entre la France et le Royaume Uni. Les comportements concernant la pause méridienne des actifs et des élèves se sont donc dé- et re-structurés dans des situations très diverses. L'étude générale reste à faire et pourra utiliser le vaste corpus international des enquêtes transport et emploi du temps. 


\section{Bibliographie}

ADEUS Agence de développement et d'urbanisme de l'agglomération strasbourgeoise (2010), Les notes de l'Adeus, mars 2010.

Anderson T., Christophersen O, Pickering K, Southwood H., Tipping S. (2009), National Travel Survey 2008 Technical Report. Prepared for the Department for Transport, UK Data Archive Study Number 5340 - National Travel Survey 2002-2010.

http://www.esds.ac.uk/doc/5340/mrdoc/pdf/5340technical_report_2008.pdf

Algava E. (2002), « Quel temps pour les activités parentales ? » Etudes et résultats, n 162, pp.1-12.

Armoogum J., Madre J.-L., Gascon M.-O., François D. (2010), « Les enquêtes nationales et locales sur la mobilité : sources et méthodes ", La revue du SOeS du CGDD, déc. 2010, pp.217-218.

Bussière Y., Armoogum J., Madre J.-L. (1996), « Vers la saturation ? Une approche démographique de l'équipement des ménages en automobile dans trois régions urbaines »" Population, vol 51, $\mathrm{n}^{\circ} 4-5, \mathrm{pp} .955-978$

Crague G. (2003), « Des lieux de travail de plus en plus variables et temporaires », Economie et Statistique, $\mathrm{n}^{\circ} 369-370, \mathrm{pp} .191-212$.

Christensen L. (2004), Busy people are hard to reach and may cause biases in a transport survey, 7th ISCTSC conference on Travel Survey Methods, Costa Rica 1.-6. August 2004. http://isctsc.let.fr/pastConf/CostaRica\%202004.html

Department for Transport (2011), NTS0101 - Trips, distance travelled and time taken per person per year, Great Britain, since 1972/73, http://www.dft.gov.uk/statistics/tables/nts0101/

Dumontier F. et Pan Ké Shon J.-L. (1999), " En 13 ans, moins de temps contraints et plus de loisirs » Insee Première, $\mathrm{n}^{\circ} 675$.

Etiévant P., Bellisle F., Dallongeville J., Etilé F., Guichard E., Padilla M., Romon-Rousseaux M. (2010), Les comportements alimentaires, quels sont les comportements? Quelles actions pour quels effets ?, Rapport d'expertise INRA, ESCO "comportements alimentaires", 64p.

Fagnani J. (1986), « La durée des trajets quotidiens : un enjeu pour les mères actives ", Economie et statistique, $\mathrm{n}^{\circ} 185, \mathrm{pp} .47-55$.

François D. (2010), "Se rendre au travail : distances et temps de transport s'allongent ", La revue du SOeS du CGDD, déc. 2010, pp.83-98.

Gallez C., Orfeuil J.-P., Polacchini A. (1997), « L'évolution de la mobilité quotidienne. Croissance ou réduction des disparités ? »RTS, $\mathrm{n}^{\circ} 56, \mathrm{pp} .27-41$.

Grignon C., Grignon Ch. (2004), « Sociologie des rythmes alimentaires » in D. Chapelot, J. LouisSylvestre (dir.), Les comportements alimentaires, Paris, Lavoisier, pp.245-257.

Guidez J.-M., Durand H, Varnaison-Revolle P., Quetelard B. (2007), " La mobilité urbaine des années 2000, Vers un découplage entre la possession et l'usage de la voiture ? ", Fiche Mobilités : faits et chiffres $\mathrm{n}^{\circ} 3$, éditions CERTU.

Hivert L. et Orfeuil J.-P. (1989), « Les déplacements domicile-travail », in Orfeuil J.-P. et Lefol J.-F. (dir.), Un milliard de déplacements par semaine. L'automobile et la mobilité des Français, Paris, La documentation française, pp.103-118.

Hilal M. (2007), « Temps d'accès aux équipements au sein des bassins de vie des bourgs et petites villes ", Économie et Statistique n402, pp.41-56. 
Hubert J.-P. et Delisle F. (2010), "L'allongement des déplacements quotidiens contribue à l'émergence d'espaces urbains multipolaires, tandis que la mobilité baisse au centre des grandes agglomérations ", La revue du SOeS du CGDD, déc. 2010, pp.49-64.

Huu Thi N. (1970), « Les repas pris hors du domicile familial », Economie et statistique, n¹4, pp.51-53. Insee (2007) Enquête nationale transport et déplacements. Instruction aux enquêteurs, Division conditions de vie des ménages, 60p.

Kaufmann V. et Flamm M. (2002), Famille, temps et mobilité : Etat de l'art et tour d'horizon des innovations, Recherche réalisée à l'intention de la CNAF et de l'Institut pour la Ville en Mouvement, 62p.

Le Breton E. (2005), « L’orientation familiale des mobilités », Netcom, vol. 19, n³, pp.172-199.

Lesnard L. (2009), La famille désarticulée. Les nouvelles contraintes de l'emploi du temps, Paris, PUF, coll. « Le lien social », 213p.

Mestdag I., Vandeweyer J. (2005), "Where Has Family Time Gone? In Search of Joint Family Activities and the Role of the Family Meal in 1966 and 1999", Journal of Family History 30(3), pp.304323.

Merlin P. (2009), L'exode urbain, Paris, La documentation Française, 170p.

Madre J.-L. et Maffre J. (1997), « La mobilité des résidants français. Panorama et évolution » RTS, $\mathrm{n}^{\circ} 56$, pp.9-26.

Madre J. -L., Axhausen K. W., Brög W. (2007), "Immobility in travel diary surveys", Transportation, 34(1), pp.107-128.

Poilot-Rocaboy G. et Kergoat M. (2006), « Pour une approche globale de l'égalité professionnelle : l'égalité des temps comme cadre d'analyse " Revue de gestion des ressources humaines, $\mathrm{n}^{\circ} 60$, pp.2-18.

Prud'homme R. (2010), " Le recul de la mobilité urbaine en France ", Transports, n 463, pp.310-315.

Ravel C. (2007), « La polarisation de l'emploi au sein des ménages de 1975 à 2002 » Economie et statistique, $\mathrm{n}^{\circ} 402, \mathrm{pp} .3-23$.

Singly F. de (1996), Le soi, le couple et la famille, Paris, Nathan, 256p.

Talbot J. (2001), « Les déplacements domicile-travail. De plus en plus d'actifs travaillent loin de chez eux ", Insee Première, $n^{\circ} 767$.

Villeneuve A. (1970), " Les déplacements domicile-travail », Economie et statistique, n¹7, pp.3-16.

Villeneuve A. (1974), "Un Français sur cinq déjeune à l'extérieur ", Economie et statistique, n56, pp.315. 


\begin{tabular}{|c|c|c|c|c|c|c|c|c|c|c|c|c|c|c|c|c|c|c|c|c|c|c|c|c|}
\hline $\begin{array}{l}\text { Heure de } \\
\text { départ tous } \\
\text { motifs }\end{array}$ & 0 & 1 & 2 & 3 & 4 & 5 & 6 & 7 & 8 & 9 & 10 & 11 & 12 & 13 & 14 & 15 & 16 & 17 & 18 & 19 & 20 & 21 & 22 & 23 \\
\hline $1973-74(\%)$ & 0,4 & 0,1 & 0,1 & 0,1 & 0,2 & 0,4 & 1,0 & 3,3 & 7,9 & 6,8 & 6,7 & 7,0 & 10,9 & 6,4 & 7,4 & 4,8 & 5,6 & 8,9 & 9,2 & 6,7 & 3,3 & 1,4 & 0,8 & 0,6 \\
\hline $2007-08(\%)$ & 0,4 & 0,1 & 0,1 & 0,1 & 0,2 & 0,3 & 0,8 & 3,6 & 8,9 & 6,9 & 5,5 & 5,6 & 8,2 & 6,0 & 7,0 & 5,4 & 7,4 & 10,7 & 9,5 & 6,2 & 3,4 & 1,7 & 1,1 & 0,7 \\
\hline
\end{tabular}

\section{Encadré 1 : Quelques questions de comparabilité entre les enquêtes nationales transports}

Cinq enquêtes nationales sur les transports ont été réalisées par l'Insee depuis 1966 en relation avec le ministère chargé des transports et, depuis la deuxième en 1973-74, I'Inrets (anciennement IRT et ONSER, devenu IFSTTAR en 2011). Seules les quatre dernières enquêtes sont exploitables.

Les quatre enquêtes ont été conçues dans une optique de série pour fournir des données de cadrage sur l'équipement des ménages en moyens de transport, l'utilisation des automobiles particulières (carnet de trajets pour un véhicule tiré au sort), la mobilité locale autour du domicile et les voyages, ou mobilité à longue distance (agenda de déplacements et de voyage pour une personne " Kish " tirée au sort). La mobilité régulière pour l'école, le travail ou la garde d'enfants en bas âge fait aussi l'objet de modules spécifiques. D’autres modules sur les coûts du transport, les voyages organisés, les communications téléphoniques, les accidents de la circulation ou l'utilisation du temps de transport... ne sont présents qu'à certaines éditions de l'enquête. Même sur les thèmes communs, les méthodologies et protocoles ont évolué mais avec le souci constant de maintenir les résultats comparables. Les collectes de chacune des enquêtes se sont toujours étalées sur douze mois chevauchant deux années, la convention est ici de les rapporter à la seconde.

Parmi les variations méthodologiques remarquables nous pouvons noter :

- l'enquête dite " 1982 » décrit les déplacements quotidiens pendant une période limitée aux mois de février, mai, juin, septembre, octobre et novembre 1981, alors que les autres enquêtes couvrent tous les mois de façon équivalente, $y$-compris pendant les vacances scolaires. En homogénéisant la période pour les autres enquêtes, nous voyons que cette restriction a assez peu d'effets sur les indicateurs globaux. Elle importe pour la mobilité des enfants mais essentiellement pour les journées sans déplacement vers l'école ;

- la limite d'âge pour les Kish a été portée à 6 ans et plus à partir de 1994, nous avons retiré des échantillons précédant, les enfants de moins de 6 ans

- l'échantillon de l'enquête de 1982 est réduit pour la mobilité des Kish : 2.905 individus contre 7.944 en $1974,14.213$ en 1994 et 18.826 en 2008. Les deux enquêtes de 1974 et 1982 ont observé la mobilité individuelle à l'aide d'un carnet de trajets auto-administré pendant une semaine. Elles ont donc livré des échantillons de journées de mobilité bien plus importants mais avec un effet de grappe. En 1994 et 2008, le protocole est celui de l'interview à domicile où l'on pose des questions sur les déplacements d'un jour et d'un week-end de référence, juste avant l'interview.

- la proportion globale de journées sans déplacement de moins de $100 \mathrm{~km}$ dans les quatre enquêtes est respectivement de $16,7 \%, 17,1 \%, 17,3 \%$ et $15,6 \%$. Ces écarts peuvent s'expliquer par les variations dans le protocole de vérification des carnets, la période spécifique de l'enquête 1982 ou encore par une extension en 2008 de la question de 1994 « vous êtes-vous déplacé(e), le... (dernier jour de semaine précédant l'interview) ? » aux sept jours précédant l'interview.

- les codages des motifs ont légèrement varié pour les lieux de repas, les lieux de travail inhabituels non codés en 1974, des déplacements de tournées professionnelles agrégés en deux déplacements en 1994 et 2008, mais pas avant, etc.

- les codes des communes de l'enquête de 1974 n'ayant pas été conservés ou codés, la définition de la mobilité locale utilisée à partir de 1982 est inapplicable (déplacements à moins de $80 \mathrm{~km}$ à vol d'oiseau 
du domicile et dans le territoire national). Pour rendre cette notion de localité associée à la mobilité d'un jour de semaine, nous utilisons pour toutes les enquêtes un critère plus simple en ne gardant que les déplacements de moins de $100 \mathrm{~km}$.

Du point de vue conjoncturel, notons aussi le développement des études supérieures après les années 1970 et les fortes variations du chômage pendant ces 35 ans. Le premier point d'observation est en 1974, juste au moment du choc pétrolier avec, alors, $3 \%$ de chômage au sens du BIT. Le dernier est en 2008, juste avant la crise financière à 7,2\% de chômage, un peu plus qu'en 1982 (6,9\% mais environ 600.000 chômeurs de moins). Enfin, le point de 1994 coïncide avec le moment de chômage maximum à près de $11 \%$ (données INSEE, taux de chômage au sens du BIT).

Le point essentiel est d'assurer la comparabilité des classes où la pause méridienne interrompt une journée de travail ou d'étude. Or ces journées correspondent à des schémas d'activité simples à noter dans les questionnaires - carnet ou agenda d'un jour -. La présence ou non de journées pendant les vacances scolaires ne devrait pas perturber les schémas d'activité de ces journées. Nous ne saurions être aussi affirmatifs pour les journées d'actifs occupés ou d'élèves sans passage sur un lieu de travail ou d'étude fixe, par exemple.

La première section de l'article utilise le module sur les habitudes de déplacements entre le domicile et un lieu de travail fixe ou un lieu d'études, ou mobilité régulière. En 1974, ce questionnement n'était appliqué qu'à une personne Kish active du ménage (2.856 individus). A partir de 1982, ces questions sont posées à tous les membres du ménage actifs occupés et scolarisés. En 2008, seul un enfant scolarisé tiré au sort est interrogé, ce qui empêche de mesurer la synchronisation du retour d'un enfant à midi avec celui d'un autre. La limitation du champ de la mobilité régulière à un seul actif en 1974 empêche, elle, de considérer toute forme de synchronisation des déjeuners dans le ménage et ne permet pas l'utilisation du module pour cette section.

L'évolution de la mobilité dépend de l'urbanisation de la zone de résidence. Nous distinguons trois grandes classes : I'agglomération de Paris, les autres grandes agglomérations et le reste du territoire, qui apparaît faiblement urbanisé par rapport aux deux autres zones. La troisième section utilise un modèle de prévision qui impose de travailler à zonage constant. Les enquêtes de 1982, 1994 et 2008 peuvent être homogénéisées mais pas l'enquête de 1974 où le code de la commune de résidence n'a pas été conservé. L'agglomération parisienne est définie par ses frontières de 1999 pour les enquêtes postérieures à 1982 et celles de 1968 pour l'enquête de 1974. Les autres grandes agglomérations sont définies par le périmètre des pôles des aires urbaines de plus de 100.000 habitants en 1999 à partir de l'enquête de 1982 et des unités urbaines de plus de 50.000 habitants en 1974. 


\section{Encadré 2 : Commentaire de l'analyse par régression logistique}

La variable à expliquer est un nombre d'aller-retour vers le lieu de travail ou d'étude habituellement supérieur à 1. Le niveau de prédiction obtenu par les modèles est élevé : proche de $75 \%$ pour les actifs employés et de $85 \%$ pour les élèves et étudiants, sauf en 2008 . Cette moindre qualité vient de l'échantillonnage des enfants scolarisés qui ne permet pas de connaître, en 2008, le comportement de plus d'un enfant dans un ménage. Malgré sa dégradation dans cette dernière enquête, l'information sur les enfants paraît encore utilisable pour analyser le comportement des actifs. En revanche, la synchronisation du retour à midi de deux enfants ou plus n'est pas une variable disponible pour l'analyse des données 2008 ni celle conjointe des trois enquêtes. Nous avons regroupé des modalités proches pour le nombre de navettes, de façon à alléger les tableaux quitte à baisser très légèrement la valeur explicative de certaines variables.

1) Facteurs explicatifs du comportement des actifs

\section{Deux variables prédominantes}

i. Synchronisation avec le conjoint. Effet très positif d'un conjoint rentrant à midi ou au foyer par rapport à un couple bi-actif ou à un actif seul.

ii. Mode de transport. Un transport à pied favorise le retour à midi alors qu'un transport en commun le contrarie. Cette variable est corrélée avec le temps de transport

\section{Deux variables importantes}

iii. Zone de résidence. Les habitants de l'agglomération parisienne rentrent moins à midi, comme les périurbains (ils n'en ont pas le temps), mais pas ceux des autres grandes agglomérations (pôles urbains des aires urbaines de 100.000 habitants et plus en 1999).

iv. Synchronisation avec les enfants. L'autre type de synchronisation compte également : effet très positif du retour d'enfants à midi mais la variable est dégradée dans l'enquête de 2008.

v. Catégorie sociale (CS 8 positions). Plus fort retour à midi des agriculteurs et artisans qui n'ont pas de cantine sur leur lieu de travail, par rapport aux employés, professions intermédiaires et cadres, moindre pour les ouvriers. La contribution de la variable baisse avec l'effectif de ces CS dans la population active.

Une variable de moindre impact

vi. Type de travail (partiel ou complet). En 1982 et 1994 les employés à temps partiel se distinguaient par un nombre plus faible de navettes, ce n'est plus le cas en 2008.

Des variables d'effet marginal

vii. Age, genre, niveau de vie et type de logement : des écarts significatifs mais très faibles.

2) Facteurs explicatifs du comportement des élèves et étudiants par ordre d'importance

\section{Une variable prédominante}

i. Synchronisation avec un autre enfant du ménage. Le fait qu'un autre enfant du ménage rentre à midi double la probabilité de le faire. On peut supposer qu'il en est de même en 2008

\section{Deux variables très importantes}

ii. Mode de transport. Il traduit l'autonomie de l'élève ou de l'étudiant et indirectement la distance.

iii. Organisation parentale. La probabilité pour un enfant de rentrer à midi est plus grande quand les parents actifs font deux navettes ou sont inactifs, et beaucoup plus faible quand le parent est isolé ou les deux parents actifs ne font qu'une navette.

Variables d'importance secondaire ou marginale

iv. Zone de résidence. L'agglomération parisienne se distingue encore en défaveur du retour à midi, même si la Ville de Paris ne suit la tendance qu'à partir de 1994.

v. Age. Les classes traduisent les différents types d'établissements d'enseignement. Cette variable joue significativement mais peu. Le changement d'établissement peut aussi occasionner une autre organisation du transport et renforcer la contribution de la variable " mode ".

vi. CS du ménage. D'un effet faible et peu lisible, la variable devient progressivement significative en suivant les enquêtes, et met en évidence les enfants d'inactifs et d'ouvriers.

vii. niveau de vie, type de logement, genre (aucun effet significatif). 


\section{Encadré 3 : Le modèle âge-cohorte}

Bien des comportements dépendent du cycle de vie, mais avec des variations à chaque génération. Un exemple simple est fourni par le revenu. II croît en moyenne avec l'ancienneté du salarié jusqu'à la retraite mais varie aussi globalement en fonction des évolutions conjoncturelles entre les périodes, de sorte qu'un salarié n'a pas le même revenu qu'un autre, au même âge et à travail comparable, dix ans plus tôt ou plus tard.

Le modèle âge-cohorte vise à séparer, dans l'évolution d'un comportement, l'effet du cycle de vie et celui de la génération. Un " profil-type » mesure la façon dont les individus se comportent au cours des étapes successives de leur cycle de vie. Les effets de cohorte sont mis en évidence par le décalage entre la trajectoire propre de chaque génération et la courbe du profil-type de la génération de référence. Ceci permet de positionner le profil-type dans une vision à long terme. Appliqué sur des prévisions de population par âge, il constitue le cœur d'un modèle prédictif pour le comportement étudié.

La construction du modèle âge-cohorte requiert une analyse longitudinale mobilisant plusieurs points d'observation, soit sur les mêmes personnes (panel), soit sur des échantillons indépendants (pseudopanel), comme dans le cas présent des enquêtes national transport. Quatre enquêtes, cependant, ne permettent de tracer qu'une partie de la courbe de cycle de vie pour chaque cohorte " $\mathrm{k}$ " réellement observée (Figure 2). Le profil-type est reconstitué pour une génération de référence par une régression sur l'âge et la cohorte.

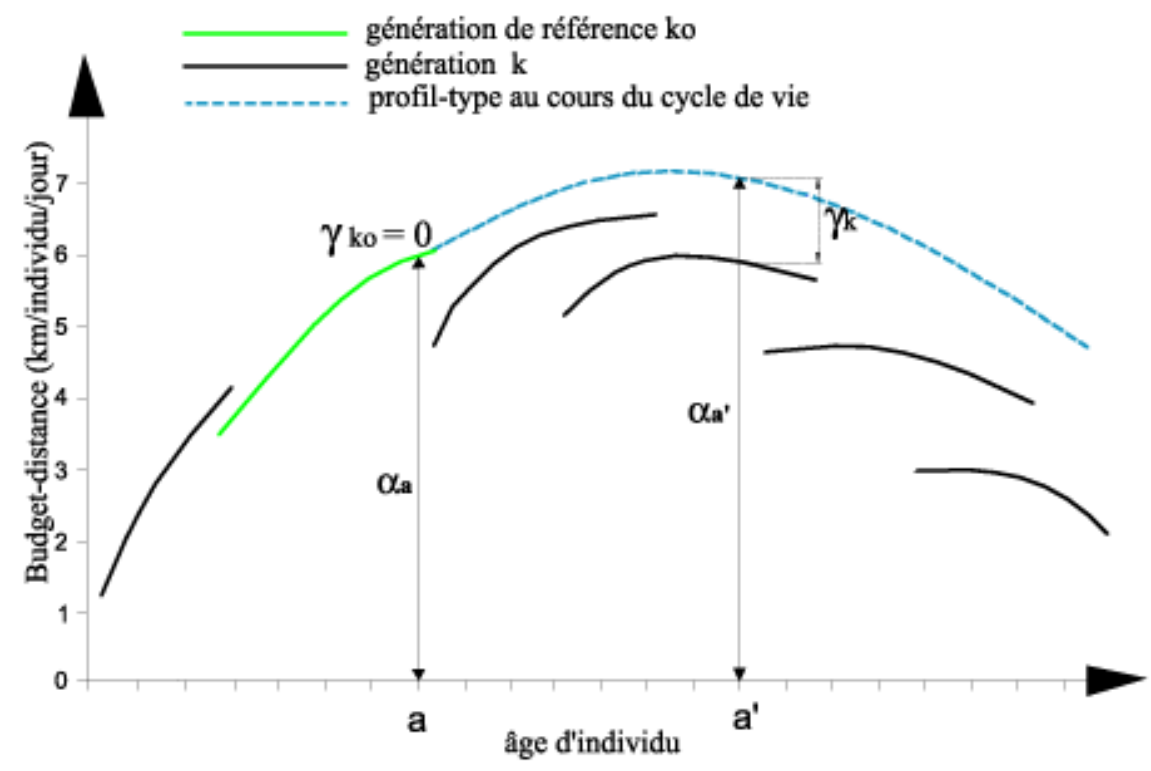

Figure 2 Principe d'estimation d'un modèle âge-cohorte (Gallez, 1994)

Dans le cadre d'un modèle âge-cohorte additif, nous faisons l'hypothèse que les trajectoires des générations successives sont parallèles. Ainsi, la formulation mathématique du modèle est la suivante :

où:

$$
V_{a, k}=\alpha_{a(k o)} A_{a}+\gamma_{k} C_{k}+\varepsilon_{a, k}
$$

$V_{a, k}$ est la mesure du phénomène observé quand l'âge de la personne est égal à $a$, sachant qu'elle appartient à la génération $k$,

- $\quad \alpha_{a(k o)}$ est la mesure du comportement à l'âge $a$ du cycle de vie d'un individu appartenant à la génération de référence $k_{o}$; on définit ainsi le " profil-type " au cours du cycle de vie, correspondant à un comportement stabilisé,

- $\quad \gamma_{k}$ est le décalage de la trajectoire de la génération $k$ par rapport à la courbe de la génération de référence $k_{o}\left(\gamma_{k}=0\right.$ pour la génération de référence $\left.k_{o}\right)$,

- $\quad A_{a}$ et $C_{k}$ sont des variables indicatrices d'âge et de cohorte,

- $\quad \varepsilon_{a, k}$ est le terme d'erreur du modèle. 